\title{
l'auscultation du barrage de serre-poncon depuis sa naissance et sur 20 ans d'exploitation
}

\author{
par \\ G. Douillet \\ Ingénieur Ecole d'Hydraulique de Grenoble \\ Chef du Service Auscultation D.T.G. Grenoble
}

et

\section{E. Ledeuil}

Ingénieur Arts et Métiers

Docteur en Mécanique du Sol

Exposé fait du Comité Français de Mécanique du Sol, séance du 23 mai 1979

\section{Introduction}

Le barrage de Serre-Ponçon, sur la Durance, a vingt ans. Lorsque en 1957 sa construction commence, il est le plus haut barrage en terre d'Europe. II fait plus de $120 \mathrm{~m}$ de hauteur et représente un volume de remblais de 14 millions de $\mathrm{m}^{2}$.

S'agissant alors pour E.D.F. d'une première, les précautions sont multipliées, l'aide de tous recherchée. M. Coyne, grand spécialiste des barrages voûtes, aux côtés des experts américains, sera écouté par E.D.F., et beaucoup d'autres personnes participeront.

E.D.F. installe un laboratoire important pour l'époque à Gap. Dans le barrage, E.D.F. prévoit la mise en place d'un réseau d'auscultation substantiel (une trentaine de capteurs de pressions, une vingtaine de piézomètres en digue. une cinquantaine dans le rocher, trois tubes à cross-arms pour mesure des tassements dans la masse et 30 repères de surface, 4 tubes avec mesures inclinométriques, de nombreux points de relevés de débits, des mesures de résistivité des eaux...)

De nombreux articles ont déjà été publiés sur Serre-Ponçon et la bibliographie sommaire est donnée en fin d'article. L'auscultation étant "vivante", après avoir rappelé certains éléments connus on cherchera surtout à les faire vivre depuis l'origine jusqu'à maintenant, c'est-à-dire parfois sur plus de 20 ans.

Le Service de l'Equipement E.D.F., Maître d'Cuvre, assure l'exploitation de l'auscultation pendant la construction, interprète et recherche les renseignements utiles pour les barrages futurs.

Après la mise en eau et la première vidange, l'auscultation est remise aux mains du Service de la Production Hydraulique qui par l'intermédiaire de sa Division Technique Générale (Service Auscultation) prend alors le relais. C'est justement là que se situe le thème de cette publication et on verra à la suite deux manières d'interpréter des mesures, les buts étant différents. II faut donc d'abord traiter des premières mesures et les analyser puis enchaîner sur l'exploitation de croisière.

Dans cette deuxième phase qui dure depuis 20 ans, l'objectif de surveillance et de sécurité est assuré dans des conditions différentes. Les responsables de l'auscultation doivent donner leur diagnostic quelles que soient les conditions :
- ils ne disposent pas de la maîtrise du plan d'eau,

- mais par ailleurs, au fur et à mesure que le temps passe. les données s'accumulent. Elles permettent alors de comprendre dans le détail, le comportement de l'ouvrage et constituent une précieuse référence tant qu'aucun désordre ne se manifeste.

Dans cette phase, la surveillance des évolutions en fonction du temps, appuyée sur des traitements statistiques rendus possibles par la taille de l'échantillon des mesures, joue un rôle essentiel.

\section{Description de l'ouvrage}

La Durance, affluent R.G. (lire Rive Gauche, par opposition à R.D., Rive Droite) du Rhone, draîne un bassin versant de près de $14.000 \mathrm{~km}^{2}$. Pour pouvoir utiliser au mieux le potentiel d'irrigation des terres et énergétique soit près de 11 milliards de kWh, il était nécessaire de créer un grand réservoir de régularisation annuelle.

Le site de Serre-Poncon qui avait retenu l'attention des Ingénieurs penchés sur le problème de la régularisation de la Durance, depuis la crue catastrophique de 1856, rassemble des aspects très positifs.

- Situé à l'aval du confluent de l'Ubaye, gros tributaire de rive gauche, le bassin versant untéressé est de $3.600 \mathrm{~km}^{2}$. - La cote du lit est encore de 665 environ à moins de 200 $\mathrm{km}$. de la mer (la cote est toujours donnée en mètres, il s'agit d'une altitude). La construction d'un barrage de 120 mètres de hauteur utile permet de créer un réservoir de 1200 millions de $\mathrm{m}^{3}$ de capacité totale.

Mais le fait que la Durance coule, dans la traversée du défilé de Serre-Poncon, sur un lit de graviers de plus de 100 mètres d'épaisseur, avait constitué longtemps pour les projecteurs un obstacle insurmontable.

\subsection{Choix du type d'ouvrage}

Rappelons brièvement les diverses considérations ayant abouti au projet définitif. En possession des résultats des reconnaissances préalables, E.D.F. a organisé, en 19491950, un concours d'idées auquel participèrent de nombreux Entrepreneurs et Bureaux d'Etudes français et étrangers. 
La question posée était la suivante : le barrage de SerrePoncon est-il techniquement possible et sous quelle forme?

L'unanimité des concurrents se prononça pour une digue en terre, structure $5 . . .-' t$ nnwa', i s'edapter aux déformations de la fondation ailuvia

\subsection{Choix du type de digue}

On disposait a priori d'une masse indéfinie d'alluvions de la Durance qui convenaient parfaitement à la réalisation de la partie perméable de la digue. On s'est donc ataché á trouver des matériaux imperméables. En deux lieux, "Lionnets" et "Molera", on est arrivé à la conclusion que l'on pouvait disposer de deux millions de mètres cubes de matériaux convenant parfaitement à la constitution d'un noyau étanche.

II était dès lors possible d'arrêter le choix sur une digue constituée par un noyau central étanche, épaulé de part et d'autre par des massifs perméables en alluvions de la Durance.

Quant au problème de l'étanchéité du lit alluvionnaire sur lequel serait construite cette digue, diverses études tant théoriques qu'économiques devaient conduire à préférer la coupure étanche verticale. Un tapis amont aurait dû faire au moins $1.200 \mathrm{~m}$ de long, bien soudé aux rives pour limiter les pertes à 400 ou 500 litres/secondes.

La solution "coupure verticale" permettait au contraire d'utiliser au mieux le site topographique, la surface du remplissage alluvionnaire ne dépassant guère $4.200 \mathrm{~m}^{2}$ au droit de l'axe de la digue. On éliminait du même coup le problème posé par la grande perméabilité du contact alluvions rocher, l'écran pouvant être soudé au rocher sain. Enfin, on tirait profit de l'anisotrophie des alluvions au point de vue perméabilité ( $\left.K / K_{v}=4\right)$. Ilétait donc normal de conclureà la solution "écran vertical".

\subsection{Description détaillée des ouvrages}

\section{- Coupure étanche}

Cette coupure a un profil en T dont la largeur, à la cote 663 est de $35 \mathrm{~m}$. Cette largeur reste constante jusqu'à la cote 653; elle diminue progressivement pour atteindre $15 \mathrm{~m}$ à la cote 643 et reste constante jusqu' au rocher qui est traité sur une épaisseur variant de 5 à $15 \mathrm{~m}$. La longueur de coupure est de $110 \mathrm{~m}$ environ à la cote 665 . Le dispositif comporte 12 lignes de forages, la largeur entre files extrêmes est de $32 \mathrm{~m}$. Il a èté ainsi réalisé une coupure étanche totale, la perméabilité des alluvions après injection étant comprise entre $10^{-t}$ et $10^{-7} \mathrm{~m} / \mathrm{s}$ soit de 500 à 1.000 fois plus faible que celle des alluvions non injectées. Ce sont les essais effectués aux plots n' 1 et 2 qui ont donné ces chiffres, montrant ainsi l'efficacité des injections. Ces plots d'essais ont montré que sur une épaisseur de 5 à $6 \mathrm{~m}$ les alluvions de surface étaient mal injectées. Afin d'asseoir le noyau sur des alluvions bien traitées, on a excavé dans la coupure étanche une tranchée de section trapézoïdale dont la largeur à la base est au minimum de $35 \mathrm{~m}$ et les talus inclinés à $2 / 1$. Cette tranchée est remplie par des matériaux imperméables compactés. Ces matériaux sont empruntés à la ballastière des Lionnets, zone des Gravas, qui fournit des matériaux dont le pourcentage d'éléments inférieurs à $5 \mathrm{~mm}$ est de l'ordre de $80 \%$. Ces matériaux fins, mis en place à une teneur en eau légèrement supérieure à l'optimum de compactage, sont compactés avec beaucoup de soin, ce qui permet d'assurer une bonne liaison entre le noyau et la coupure.

\section{- Digue}

La digue est en terre et en alluvions compactées,

La projection en plan de l'axe de l'ouvrage, se présente sous la forme d'une ligne brisée (angle de $150^{\circ}$ ). La branche rive droite coupant les alluvions dans la partie la plus resserrée du défilé, alors que la branche rive gauche s'appuie sur le flanc amont de l'éperon rocheux du Serre de Monge.

Le développement en crête, à la cote 788 , est de l'ordre de $600 \mathrm{~m} . \mathrm{La}$ digue est constituée par un noyau étanche en matériaux imperméables, enserré entre deux recharges en matériaux au moins 100 fois plus perméables. Son cube total est évalué à $13.400 .000 \mathrm{~m}^{3}$ dont $1.700 .000 \mathrm{~m}^{3}$ pour le noyau. Dans l'axe de l'ouvrage, le noyau a $8 \mathrm{~m}$ de large à la cote 784 et environ 55 màl'assise; ses deux talus, emprisonnés dans les recharges, ont un fruit de 1 pour 5 , sauf dans la zone du Serre de Monge.

Compte tenu des caractéristiques différentes des fondations oủ s'appuie le noyau et, afin d'éviter qu'il ne se fissure sous l'effet des tassements différentiels, les matériaux le constituant sont mis en place à une teneur en eau supérieure de 1 à $2 \%$ à l'optimum de compactage, afin de leur donner une certaine plasticité.

Les recharges sont constituées par des matériaux alluvionnaires provenant du lit de la Durance.

Les fruits des talus des recharges sont de :

- 3,1 entre les cotes 666 et 700 à l'amont et 666 et 706 à l'aval

- 2.5/1" " 700 et $780^{\circ " ~ " ~} 706$ et $740 "$

- $2 / 1 . \quad 780$ et $784 "$ " 740 et 780 "

- $1,75 / 1$ * * 784 et 788 " . 780 et 788

Des zones de transition, tant à l'amont qu'à l'aval du noyau ont $20 \mathrm{~m}$ de largeur en bas et 4 en haut, elles sont en alluvions riches en fines, extraites à sec.

Le drain sous la recharge aval a $10 \mathrm{~m}$ d'épaisseur, il est réalisé avec des alluvions extraites dans l'eau courante possédant moins de $2 \%$ d'élements inférieurs à 80 microns. II ne sera jamais concerné par l'eau en phase d'exploitation.

\section{- Voile d'étanchéité et de drainage dans la fondation rocheuse}

Pour $4.000 \mathrm{~m}^{2}$ de coupure étanche à réaliser dans les alluvions et $50.000 \mathrm{~m}^{2}$ de section droite fermée par le noyau, il a fallu prévoir un traitement de rocher sur $90.000 \mathrm{~m}^{2}$. Un tel traitement a été réalisé en totalité à partir des 2 galeries d'injection R.D. et R.G. plus l'usine.

\section{Description et justification du dispositif d'auscultation}

\subsection{Présentation}

L'ensemble des instruments de mesure prévus pour l'auscultation de la digue doit permettre d'observer, à partir d'un certain nombre de points bien déterminés, le comportement de la digue pendant la construction, lors de la première mise en eau, puis durant toute la vie de l'ouvrage. Par ailleurs, les instruments ont été implantés de manière à réduire, dans la mesure du possible, la gêne apportée à l'entreprise au cours des travaux. L'ensemble de ces instruments répond aux préoccupations concernant les points suivants

- Percolation de l'eau et surface de saturation soit à travers la digue et les fondations soit dans la recharge amont dans le cas d'une vidange de la retenue.

- Efficacité de la coupure étanche et du noyau imperméable.

- Tassements et déformations du noyau, des alluvions injectées (coupure étanche), de la recharge aval et suivi de la consolidation du noyau au cours du temps.

- Pressions interstitielles et pressions totales développées dans le noyau.

- L'utilisation de plusieurs types de cellules, cellules piézométriques du "Bureau of Reclamation" àtransmission par fluide, cellules électriques de pressions interstitielles et pressions totales, permet de comparer les observations enregistrées et d'obtenir ainsi de meilleurs résultats par élimination de lectures anormales. On peut aussi classer ces appareils en fonction de leurs qualités et défauts respectifs.

\subsection{Localisation des appareils}

Les deux vues en plans de la figure 1 situent un certain nombre de ces appareils.

Le lieu des verticales passant par l'axe du sillon rocheux possède à lui seul la presque totalité des instruments : 
REPERES TOPOGRAPHIQUES TUBES DE DEFORMATION

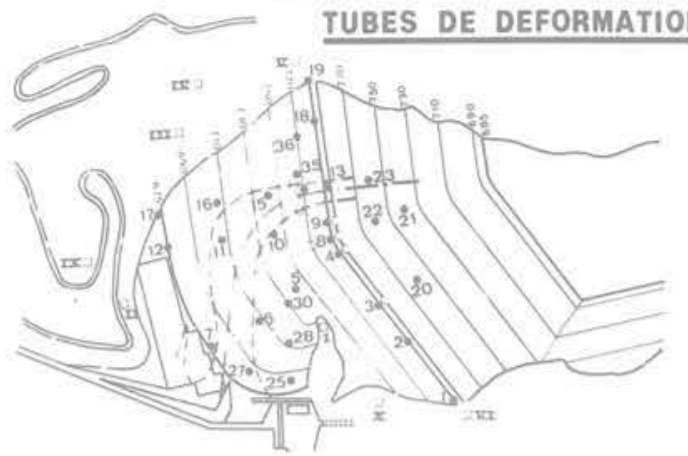

Reperes topographiques

$\square$ Piliers d'observation

Tubes de deformation

Ligne de niveau 630 (Rocher) Axe du sillon rocheux

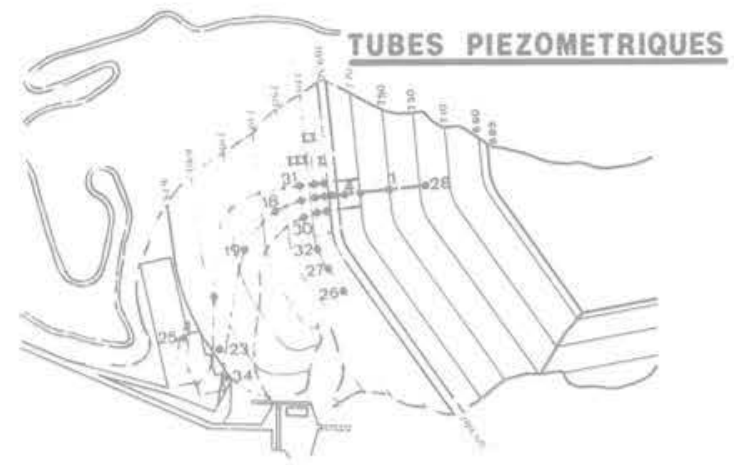

Fig. 1

- cellules électriques et à circulation d'eau, réparties dans le noyau suivant les 16 points d'un seul plan transversal.

- 18 prises de pressions par tubes piézométriques plus

10 prises de part et d'autre de l'axe du sillon.

- 50 piézomètres dans le rocher (essentiellement en R.G.).

-3 repères de tassement.

La surface de la digue est suivie par 31 repères topographiques examinés depuis un réseau de stations matérialisées initialement par 9 piliers et étoffé par la suite. En crête, 10 repères de nivellement complètent le dispositif.

La zone de la "brisure" de la digue a été suivie par un piézomètre équipé en tube de déformation et par 3 tubes de déformation dans le noyau, placés deux de part et d'autre de la brisure et un dans l'axe de la brisure. Enfin, un tube de déformation devait permettre de voir l'influence du Serre de Monge sur le noyau.

\subsection{Description sommaire des appareils :}

\section{- Repères superficiels}

lis doivent éviter d'être influencés par l'effet de peau (fluage superficiel du matériau) pour cela ils sont ancrés à plus de $2 \mathrm{~m}$ de profondeur avec une semelle de $2 \mathrm{~m}^{2}$.

\section{- Repères de tassement internes}

Ils ont pour but de dèterminer les tassements en différents points intérieurs au barrage. Il y a 3 files verticales de points de mesures, repères T 13, T 14, et T 15. La cote des "cross arms" est lue par rapport à la surface par une "torpille" possédant des cliquets montés sur ressorts et capables de se verrouiller au contact de la plaque rigide du fond (pour description voir référence 11). La cote du tube à la surface est donnée par mesure topographique.

\section{- Cellules à pression de terres}

- 3 cellules W.E.S. - (Waterways Experiment Station à Vickburg (U.S.A.) ; un coussin liquide (du mercure) trans- met les efforts du sol sur la plaque supérieure de la cellule à une membrane sous laquelle sont collés 4 straingages. Les déformations de cette membrane de 4 à $5 \mathrm{~mm}$ d'épaisseur (suivant la précision désirée et la charge maximum) sont suivies par la variation de résistance des straingages. Un étalonnage a permis de lier linéairement ces variations de résistance à la charge sur la cellule.

- 10 cellules Telemac : les cellules Telemac construites d'après un brevet "Coyne" sont basées sur les lectures de variation de fréquence d'une corde vibrante. Pour les contraintes de sol, la corde est tendue d'autant plus que la pression est plus forte. Deux plaques face à face possèdent à leur partie interne des petites biellettes capables de transformer un mouvement de rapprochement des plaques en une traction du fil.

\section{- Cellules de pressions interstitielles}

- 12 cellules U.S.B.R. Leur principe est très simple. Une pierre poreuse permet la transmission de pression entre les eaux contenues dans la terre et de l'eau contenue dans deux tuyaux sortant de la digue et munis chacun d'un manomètre.

La pression lue, corrigée de la hauteur géomètrique de la cellule (tassement compris) par rapport au poste de lecture est la pression interstitielle au point considéré.

- 3 cellules W.E.S.: Leur principe est le même que pour les pressions de sol, une grille empêche le sol de déformer la membrane mais permet à l'eau de la déformer.

- 9 cellules Telemac : le principe est ici quelque peu différent de celui des pressions de sol. La corde est tendue entre deux membranes (plaques de 3 à $5 \mathrm{~mm}$ d'épaisseur en laiton, suivant la sensibilité et la plage de lecture désirée) qui détendent la corde lorsque la pression augmente.

\section{- Tubes piézométriques}

Dans les zones perméables des trous forés par battage (à l'eau lourde ou à l'amidon) sont équipés de tubes étanches sur toute leur hauteur sauf sur leurs 2 derniers mètres en bas où ils sont crépinés. Les dix derniers mètres sont forés avec de la boue à l'amidon (au moins 40 à $50 \%$ d'amidon, le reste en bentonite). A la fin du forage, le tube est descendu. du sable est déposé sur 4 ou $5 \mathrm{~m}$ de haut autour du crépinage, puis un blocage étanche est réalisé avec un mélange d'argile et de ciment. Ensuite, le tube est lavé à l'eau claire par circulation, enfin des bactéries sont déposées au fond du tube. Ces bactéries en se développant digèrent l'amidon. permettant de recréer au niveau du crépinage une perméabilité suffisante.

\section{Tassements}

4.1. Les tassements ont été suivis dès le stade de la construction. Sur la figure 2 nous voyons l'allure des tassements de chaque point ausculté, repéré soit par son altitude, soit par le numéro du repère. Durant l'hiver, soit pendant l'arrôt des mises en remblais la pente des courbes diminue nettement pour reprendre dès la reprise des chargements. Une des premières études faites à partir de ces tassements a été de définir la surhauteurà donner à la digue pour que, en fin de construction et même longtemps après, la crête reste plus haute au centre de la digue que sur les rives. Alors que les prévisions de tassements issues de l'extrapolation des mesures conseillaient une surhauteur de $0,4 \mathrm{~m}$, l'Ingénieur Conseil Américain a porté ce chiffre à 1,5 $\mathrm{m}$ pour une question d'esthétique voulant le barrage plus haut au centre.

4.2. Les matériaux du noyau ont souvent une bonne ossature, soit 30 ou $50 \%$ d'éléments supérieurs à $5 \mathrm{~mm}$, ce qui leur donne une répartition de $36 \%$ de tassement réputé instantané pour seulement 20 à $30 \%$ de tassement hydraulique.

Le repère de tassement " $\mathrm{T} 13$ " situé dans le noyau permet aussi de suivre l'évolution $h$ de la consolidation de chaque tranche de $\mathrm{h}=15 \mathrm{~m}$ de terre située entre 2 "cross-arm" consécutifs. 
T133

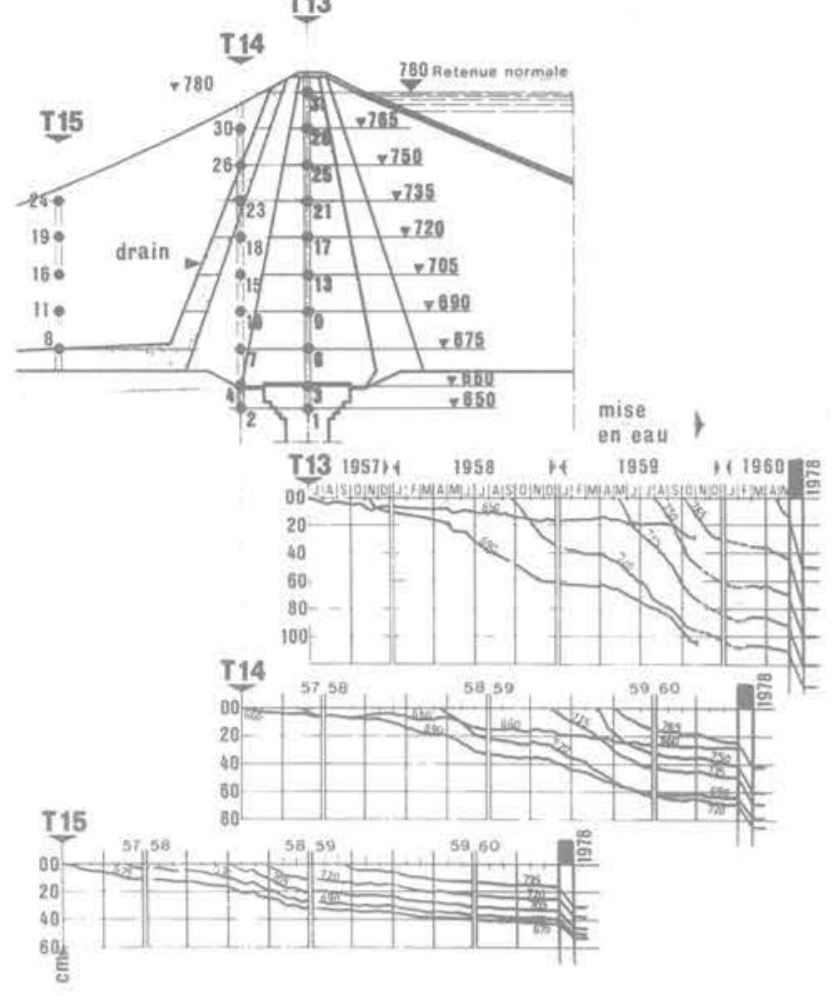

Fig. 2 Repères de tassement (profil principal)

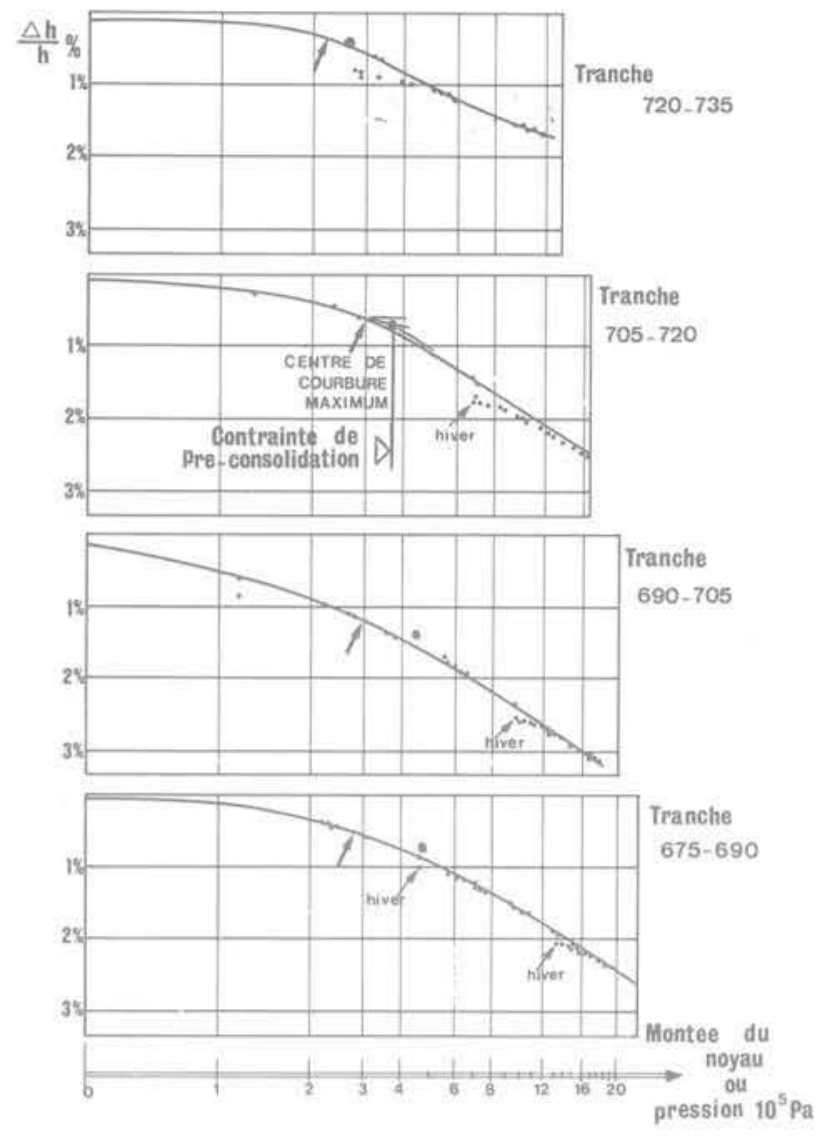

Fig. 3
La figure 3 donne l'allure de l'évolution de $\Delta h / h$ en fonction de la charge située au-dessus. Le palier d'hiver (trop court) perturbe cette courbe, tout en ne permettant pas de prendre en compte un état ultime de consolidation.

Une tangente à la courbe vers $1510^{5} \mathrm{~Pa}$ donne une idée du module œdométrique E' sous cette charge, il se situe entre 1 et $1,510^{8} \mathrm{~Pa}$. Ce chiffre place le noyau dans un domaine de terre argileuse avec déjà une bonne ossature (nous pouvons rappeler que les essais de laboratoire donnaient des chiffres nettement plus faibles, soit 4 à $610^{7} \mathrm{~Pa}$ ). Si l'on associe ce module au Cv calculé dans le noyau, de l'ordre de : $0,1 \cdot 10^{-4} \mathrm{~m}^{2}\left(\mathrm{a} 0,9.10^{-4} \mathrm{~m}^{2} / \mathrm{s}\right)$, on peut retrouver une estimation de la perméabilité $K$ :

$\mathrm{C}_{\mathrm{v}}=\mathrm{K} \cdot \mathrm{E}^{\prime} / \mathrm{W}$

d'où $K=0,1 \cdot 10^{-4} \cdot 10^{3 / 3} 1 \cdot 10^{8}=10^{-10} \mathrm{~m} /$ à $10^{-9} \mathrm{~m} / \mathrm{s}$

Ces quelques chiffres tirés des mesures d'auscultation bien en accord avec les essais de laboratoires permettent de vérifier que la mise en place a bien répondu à nos désirs. L'auscultation n'est pas que la mesure de déplacements, elle permet d'analyser la matière elle-même.

4.3. Les tassements (consolidation de chaque tranche) tracés en fig. 3 . avec les pressions en abscisse sur une échelle logarithmique permettent aussi de percevoir une certaine préconsolidation due au compactage. La construction classique de Casagrande permet de dire que le matériau du noyau compacté avec un rouleau à pneu gonflé à 6 ou $6,510^{5} \mathrm{~Pa}$, a imprimé au matériau une contrainte de pré-consolidation de 4 à $4,510^{5} \mathrm{~Pa}$.

Un tel enseignement est intéressant puisqu'il indique une qualité de compactage, et si on le compare aux essais de laboratoire où l'on trouve souvent moins de $0,510^{\mathrm{s}} \mathrm{Pa}$ de pré-consolidation (échantillons non remaniés), on peut juger de la valeur comparative de tels essais. Pour les esais de laboratoire sur échantillons remaniés, on a plus souvent 1 à $410^{5} \mathrm{~Pa}$ de pré-consolidation.
Les conséquences de cette contrainte de pré-consolidation se retrouveront dans les tassements car nous trouvons : $3 \%$ dans le noyau (donc en place).

5 à $6 \%$ pour les essais remaniès,

6 à $8 \%$ pour les essais non remaniés.

La transposition des essais de laboratoire aux réalités sur le chantier est donc très délicate comme on le voit ici. Mais imprimer au sol une telle contrainte de pré-consolidation indique qu'un remblai de $20 \mathrm{~m}$ de haut compacté de cette manière ne doit pas tasser. Nous verrons même qu'une certaine décompression peut avoir lieu avec le temps.

4.4 Pendant l'exploitation de l'ouvrage les tassements ont continué et se poursuivent toujours (fig. 2). Ils sont liés principalement au noyau et aux alluvions du sillon et très faiblement aux recharges.

Leur traduction à la surface de l'ouvrage est illustrée sur la figure 4 oủ l'on constate que les vitesses actuelles présentent une organisation dans l'espace très voisine de celle observée aussitỏt après la mise en eau : le noyau qui se tasse encore de $5 \mathrm{~mm} /$ an sur son axe est le seul lieu de tassement important. Ce tassement concerne essentiellement les couches inférieures de matériaux (la presque totalité dans la moitié inférieure du noyau, soit par an $0,1 \%$ de la hauteur de matériaux intéressée) alors qu'une très légère tendance à la dilatation semble au contraire intéresser les $10 \mathrm{~m}$ supérieurs. Plus à l'aval la prédominance du tassement des alluvions naturelles du silion sur celui des matériaux des zones de transition et des recharges est très nette: I'ampleur de ces mouvements depuis 15 ans, soit 5 à $8 \mathrm{~cm}(0,5$ à $0,8 \%$ de l'épaisseur de la fondation alluviale) est à comparer à celle acquise pendant la période de construction et des premières mises en eau, soit 45 à $60 \mathrm{~cm}$ en 5 ans. ( 45 à $6 \%$ de la fondation alluviale).

La parfaite continuité dans le temps de ces mouvements depuis l'origine et la modicité des vitesses actuelles sont les caractéristiques qui permettent d'assurer qu'aucun phéno- 


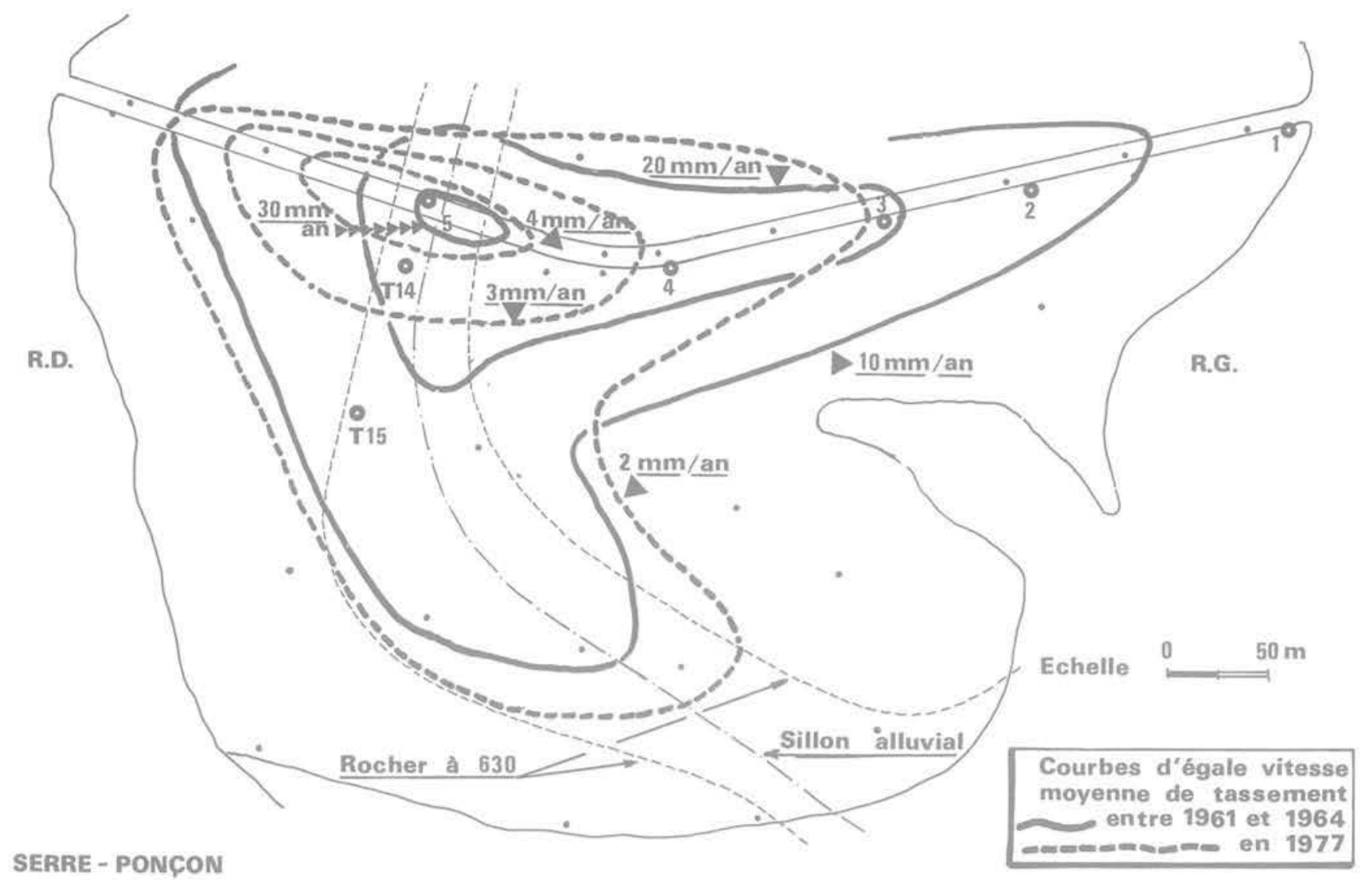

Fig. 4 Déplacements verticaux irréversibles

mène parasite n'est apparu, qui aurait pu être générateur de désordre dans les matériaux du corps du barrage et de sa fondation. Enfin, une certaine influence de "déjaugeage" du barrage par le remplissage de la retenue est perceptible, mais îl est difficile d'assurer qu'il s'agit d'un mouvement réversible ou seulement d'un soulagement momentané du tassement général.

\section{Déformations horizontales}

Initialement il était prévu de suivre les déformations non seulement à partir des repères de surface, dont il va être question, mais aussi sur quatre verticales matérialisées par quatre tubes plastiques mis en place dans des forages et scellés. Dans ces tubes on descendait une sonde "Eastman" comprenant pendule, boussole et caméra permettant de définir la déformation du tube en amplitude et direction. On pensait pouvoir ainsi être éclairé sur les déformations dans la zone de cambrure de la digue et la partie du noyau appuyée sur le Serre de Monge. En fait, malgré un dépouillement minutieux, il n'a pas été possible de définir des déplacements à mieux que $10 \mathrm{~cm}$ à $100 \mathrm{~m}$ de profondeur, ce qui est, bien entendu, totalement insuffisant et a conduit à abandonner immédiatement ce type d'investigation.

On voit sur la figure 5 le dispositif qui a permis, depuis l'origine, de suivre les déplacements de surface, conséquences des déformations internes de la structure. Les extrémités des visées issues du réseau d'observation extérieur au barrage sont indiquées pour chaque repère, ainsi que les ellipses qui, autour de chacun d'eux, définissent le domaine à l'intérieur duquel il y a une probabilité de $87 \%$ pour que la précision des mesures d'angle situe le point. Ce domaine ne dépend que des caractéristiques géométriques de la triangulation, de l'hypothèse retenue pour la fixité des piliers du réseau et de l'écart moyen quadratique lié à la précision des mesures d'angle, soit dans le cas de Serre-Poncon, compte tenu des conditions de visée (lonqueur, rasance, etc.) 3 secondes centésimales. On observe que la longueur des axes des ellipses ne dépasse pas $15 \mathrm{~mm}$, ce qui garantit, dans les conditions normales, une dètermination largement suffisante pour un ouvrage de cette importance.

\subsection{Composante horizontale des mouvements de convergence des matériaux vers l'axe de la vallée}

Induite par le tassement, cette composante s'est, depuis la première mise en eau, très systématiquement organisée par rapport à l'axe de la vallée sans aucune anomalie spatiale ni temporelle. Les mesures, commencées dès la mise en place des repères, assurées aussitôt que l'avancement du remblai le permetait, ont montré des amplitudes inférieures à $20 \mathrm{~m}$ au cours des six premiers mois et un amortissement très régulier sur les huit premières années d'exploitation. La figure 6 met en évidence l'organisation par rapport à l'axe de la vallée des déplacements totaux depuis l'origine et de la vitesse moyenne actuelle qui n'évolue plus quère. La seconde est approximativement la moitié de la première. La figure 7 représente la courbe d'amortissement du mouvement horizontal R.D. - R.G. de 2 repères placés sur la crête en rive droite. C'est dans cette zone que l'on constate le gradient de déplacement horizontal le plus important. C'est le seul point ou l'on note un allongement d'ampleur notable: la base de $35 \mathrm{~m}$ correspondante s'est allongée de $35 \mathrm{~mm}$ depuis l'origine, soit $1000.10^{-6}$, alors qu'elle a actuellement un allongement relatif de $40.10^{-6}$ par an. Partout ailleurs on note des allongements extrêmement faibles ou des contractions qui se poursuivent à l'heure actuelle avec des vitesses de 20 à $50,10^{-6}$ par an.

\subsection{Déplacements dans la direction amont-aval}

II avait été noté dès l'origine que les déplacements amont aval étaient nettement inférieurs à ceux observés sur des 


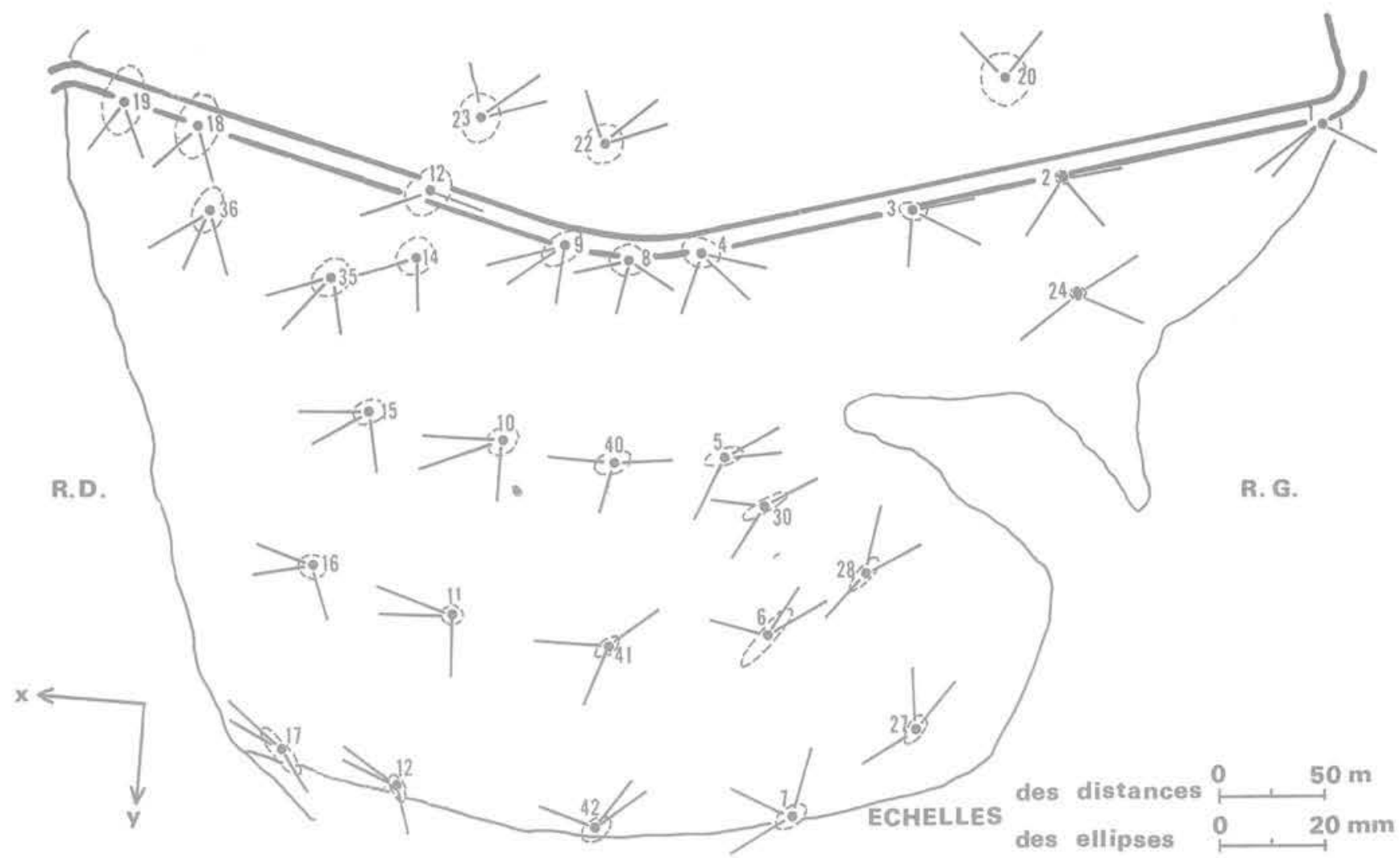

SERRE - PONÇON

Fig. 5 Planimétrie. Ellipses d'incertitude de détermination des repères

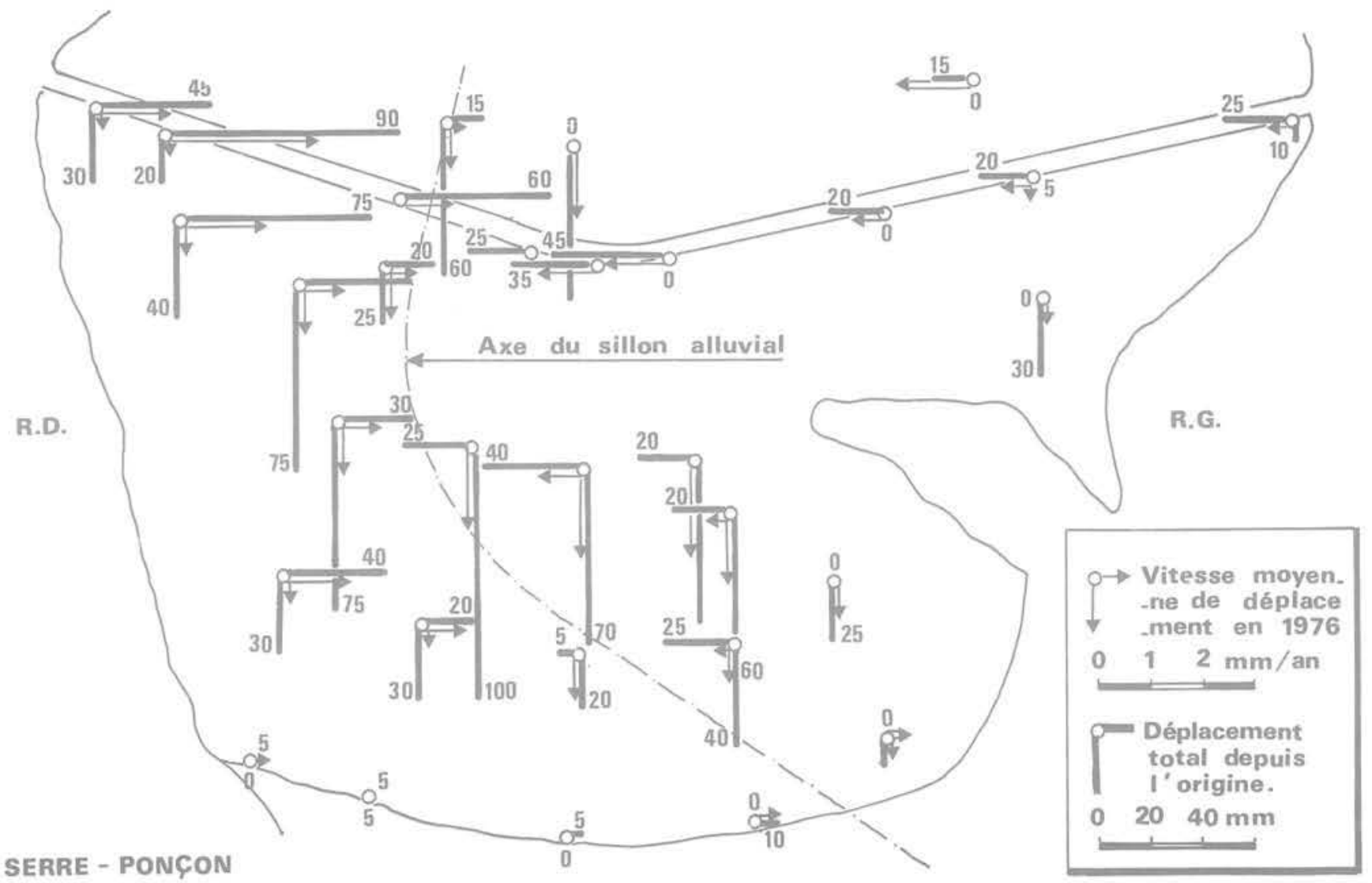

Fig. 6 Déplacements horizontaux irréversibles 
ouvrages sumilaires, et que dans cette direction le noyau avait un comportement élastique vis-à-vis des variations de chargement créées par les modifications de plan d'eau au cours des premiers remplissages.

L'exploitation des mesures faites au cours de l'ensemble des cycles d'exploitation depuis 1960 et correspondant à des cotes de retenue variables a permis de préciser ces éléments et notamment d'évaluer avec rigueur la part réversible qui revenait, dans les déplacements observés, au fonctionnement élastique de la structure sous l'effet des variations de charge hydraulique et celle, irréversible, correspondant aux évolutions affectant les matériaux constitutifs.

On a utilisé, pour cela, la méthode, maintenant et depuis de nombreuses années, systématiquement utilisée pour traiter toutes les grandeurs périodiquement relevées en vue de l'auscultation des grands barrages d'E.D.F. Maintes fois déjà exposées dans diverses publications : il s'agit d'un ajustement, par la méthode des moindres carrés, de l'échantillon (le plus large posible) de mesure d'un appareil, sur une formulation mathématique simple modélisant les comportements réversible et irréversible.

Les déplacements hydrostatiques réversibles ainsi déterminés pour une montée du plan d'eau de 740 à 780 soit $1 / 3$ de la hauteur d'eau totale sont représentés sur la figure 8 . Il est confirmé qu'ils sont de très faible amplitude et qu'ils intéressent essentiellement la zone centrale de la partie supérieure de l'ouvrage. Les mouvements irréversibles qui se sont produits depuis l'origine sont représentés sur la figure 6 ou apparaissent simultanément le déplacement total depuis l'origine et la vitesse moyenne actuelle. Ils sont tous dirigés vers l'aval. Très faibles sur les rives et nuls au centre pour la crête, ils sont maximums (comme on l'observe dans la plupart des digues en terre) à mi-hauteur de la recharge aval : $1,5 \mathrm{~mm} /$ an pour un déplacement total de 60 à $100 \mathrm{~mm}$. Dans tous les cas, les amplitudes sont très réduites. La figure 9 montre la régularité de l'amortissement du mouvement sur deux repères situés près de l'axe de la digue.

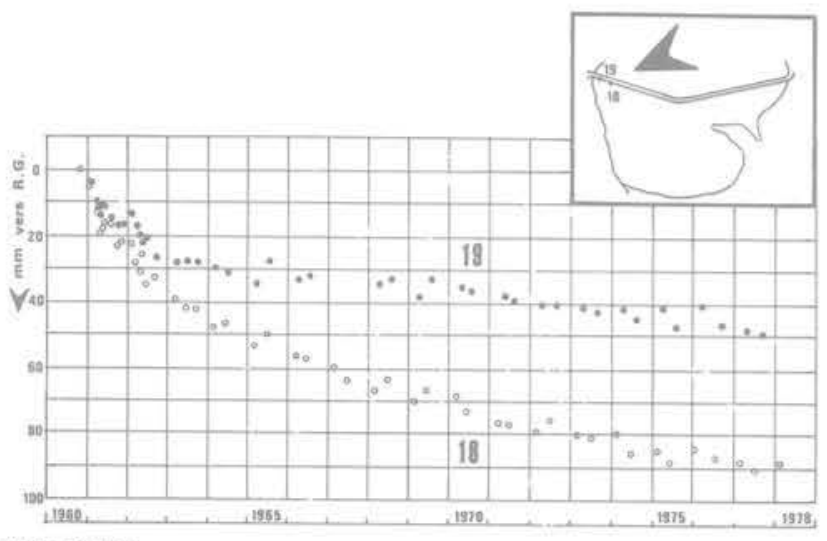

SEnHE PONGON

Fig. 7 Amortissement du déplacement R.D./R.G. des repères 18 et 19 en R.D. de la crète de 1960 à 1978

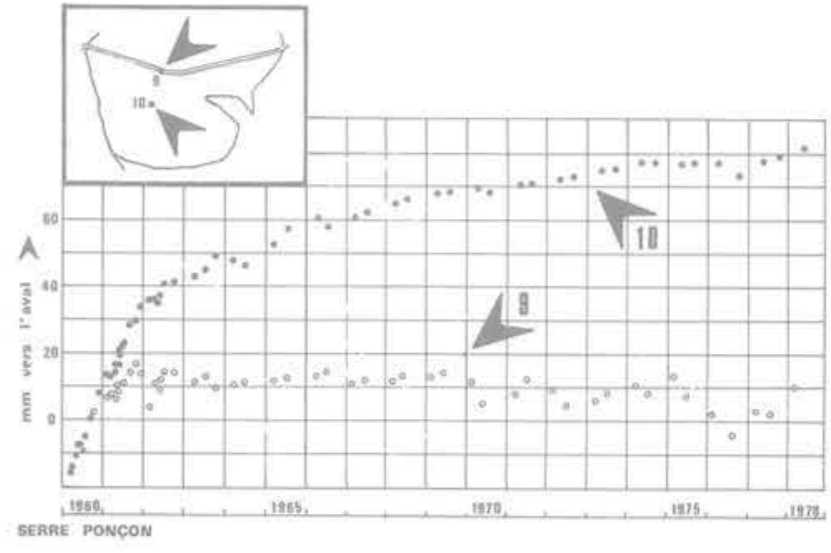

Fig. 9 Déplacement amont-aval des repères 9 et 10

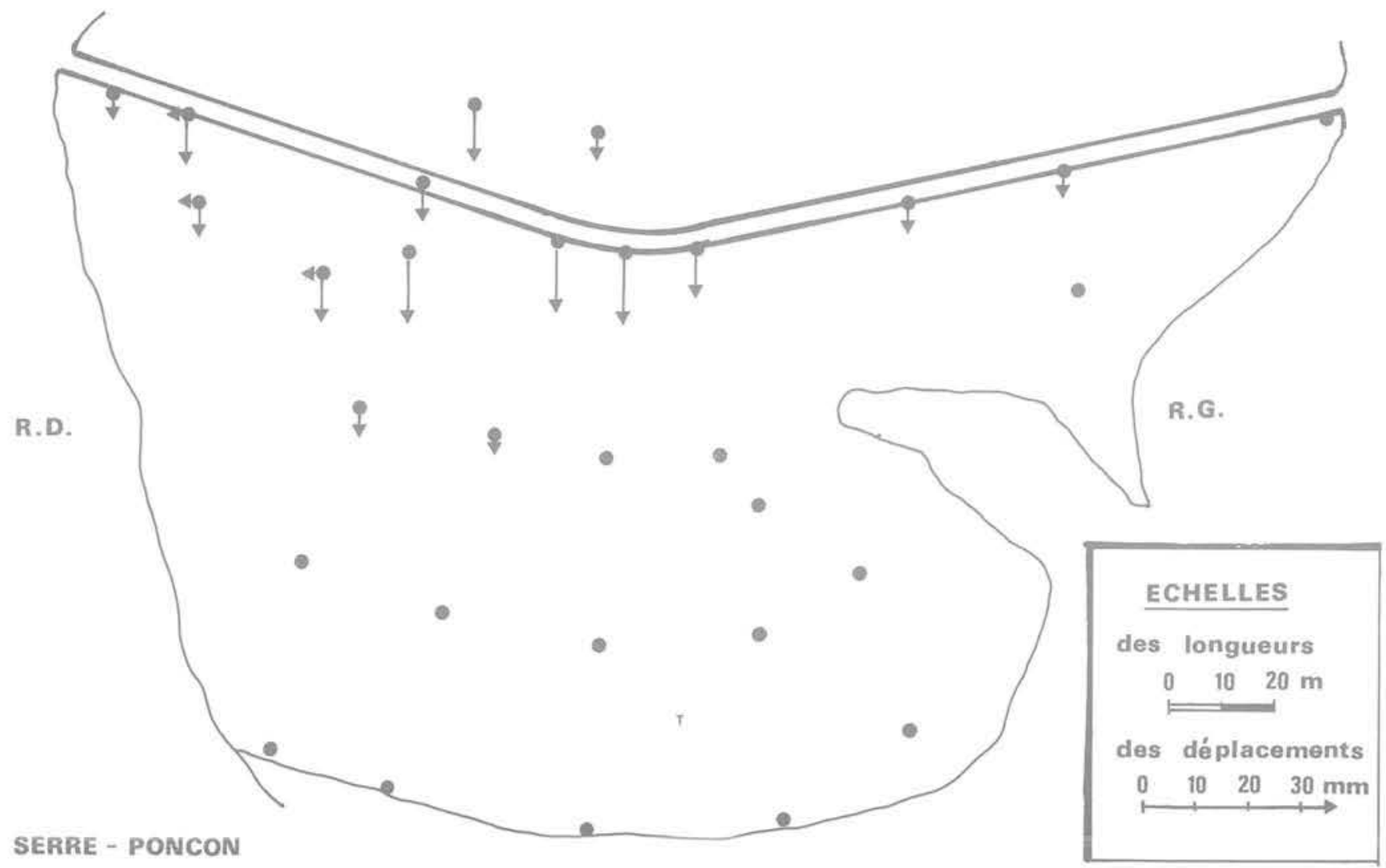

Fig. 8 Déplacements hydrostatiques réversibles pour une montée du plan d'eau de : 740 à 780 


\section{Comportement hydraulique}

\subsection{Le noyau}

Dans le noyau, les divers appareils de mesures de pressions, et les courbes de lectures données en figures 10 et 11 ont été souvent décrits. Avant d'aborder le comportement hydraulique, quelques remarques concernant les pressions de sol peuvent être faites. On peut, dans la figure 10, remarquer le décrochement pour la cote de remblais 773 qui en fait correspond à la première phase de mise en eau (soit 40 m)... En ce qui concerne la cote 732 la baisse des pressions de sol peut être imputée à des effets de voûte... A la base (cellule 132) les effets de voûte sont peu sensibles étant donné peut être la grande largeur du noyau, mais il n'en est plus de même au-dessus de la cote 735 oú près de $50 \%$ des charges supérieures sont retenues. Tout ceci prouve que le souci de compactage du côté humide, afin d'améliorer la plasticité du noyau vertical, était et reste toujours dans de tels barrages en terre une ardente obligation. En ce qui concerne les pressions interstitielles données en figure 11 , jusqu'à 10 métres de remblais $100 \%$ de la charge sont repris par les pressions créées. On peut voir la dissipation d'hiver lors de l'arrêt des chargements et la reprise des mises en pression avec la reprise des remblais. La mise en eau, ayant causé des tassements profonds non suivis en totalité par la surface, tel que décrit ci-dessus, est enregistrée par une baisse des pressions surtout au milieu du noyau. La cellule 415 montre une excellente corrélation avec la charge et atteste d'un excellent comportement du haut de la coupure étanche. Actuellement, la baisse de 15 à $20 \%$ corrélative d'une diminution du débit global de percolation indique vraisemblablement un colmatage amont, recoupé par d'autres observations par ailleurs.

\section{- Images des écoulements instantanés}

A partir de nombreuses mesures faites pendant la construction, on a cherché à tracer un réseau de lignes équipotentielles c'est-à-dire avec $\Delta H=\mathrm{Ct}=5 \mathrm{~m}$. On a beaucoup de mesures, mais peu de points de mesures. Quelques remarques permettent de multiplier sans un trop grand risque d'erreur, le nombre de points oủ le potentiel est déterminé. - A l'exécution la limite latérale du noyau a toujours été très stricte, elle se situe dans une bande de $50 \mathrm{~cm}$ et les transitions nettement plus perméables ont été bien suivies. On peut alors considérer une coupe transversale du noyau oủ les cellules placées de 1,25 à $2 \mathrm{~m}$ seulement du bord sont significatives et permettent avec la cellule centrale de définir une répartition transversale conforme aux courbes de Taylor (consolidation en fonction de la profondeur et du facteur temps).

Comme indiqué dans le schèma (fig. 12), les risques d'erreur sont faibles. L'équilibrage des pressions est pratiquement obigatoire, car un état local de surpression ne peut se concevoir longtemps, puisqu'il y aura écoulement vers les zones de potentiel plus faibles. La figure 14 propose les courbes adoptées pour un niveau (732) du noyau et à 2 époques, pendant la phase dite active de montée du remblai et après le repos d'hiver, donc la dissipation des pressions. Après avoir multiplié les points transversalement, on peut aussi les multiplier dans le sens de la hauteur par interpolation et extrapolation puisque, en phase active, ce qui se passe avec $x$ mètres de remblais de plus peut être confondu avec la lecture que l'on a eue avec une cellule placée à $x$ mètres de profondeur. Les profils (fig. 13), permettent de trouver des lignes de répartition continues et de dresser les répartitions transversales.

Une telle méthodologie décrite, on peut tracer pour n'importe quel niveau de remblais une "image instantanée" des répartitions de pressions interstitielles dans le noyau.

On obtient des courbes équipressions, lesquelles, en tenant compte de l'altitude $z$ de chaque point, se transforment en réseau de courbes équipotentielles. Dès lors nous pouvons, en traçant le réseau orthogonal faisant des "petits carreaux", définir les lignes de courant représentant le régime d'écoulement instantané. Bien sûr en réalité l'eau ne suivra pas de telles lignes car les pressions se modifient, mais cette image instantanée permet de voir vivre le noyau du barrage.
- Particularités des écoulements décelables pendant la construction

On peut observer:

- la bonne homogénéité des figures donc vraisemblablement les matériaux du noyau sont homogènes et bien compactés.

- en phase active (reprise de la montée du remblai), les fortes pressions créées (près de $100 \%$ de la charge en surface; cf. la figure de corrélation 13) permettent de voir, dans les images d'écoulement, de l'eau qui se dirige vers le haut et de l'eau qui va réalimenter l'horizon desséché durant l'hiver précédent. Ces deux phénomènes disparaissent ou presque après un nouvel arrêt d'hiver (fig. 14).

- en ce qui concerne la coupure étanche, les pressions de l'eau du noyau repoussent les percolations amont-aval 10 mètres sous la ligne piézométrique aval, alors que la charge est de 18 mètres par le seul effet de la dérivation de la Durance.

- la reprise de nouveaux chargements après chaque arrêt d'hiver amène la reprise de l'écoulement des eaux issues de la consolidation (fig. 14).

\section{- Ecoulement pendant la première mise en eau}

- la mise en eau se fait sentir d'une façon claire et progressive, on peut voir un complément de saturation des zones desséchées par l'arrêt d'hiver (perte de tubes de courant) qui disparaitra après quelques mois. Pour la coupure étanche, il semble que le contact à la base du noyau ait été soumis au contraire à une nouvelle phase de consolidation par création de tubes de courant dans la masse du matériau, le débit sortant à l'aval étant plus grand que celui entrant (fig. 15).

Même si les images citées en $\S 6.1$ ne sont qu'instantanées, il n'en reste pas moins qu'un écoulement quasi-permanent existe avec des gradients variables. Puisque, dans chacun de ces tubes d'écoulement il passe un dëbit calculable avec les données, pour la totalité du noyau 1 litre/seconde pour les $50.000 \mathrm{~m}^{2}$ de surface doit être un maximum.

\section{- Régime en exploitation}

La définition des caractéristiques précises du régime en exploitation se heurte à la difficulté suivante : d'une part la cote de retenue est constamment variable, d'autre part les phénomènes hydrauliques, dont le noyau est le siège, sont affectés par des "retards" importants à l'échelle de celle des variations de cote. Dans ces conditions, un résultat de mesure à une date donnée doit être rapproché non seulement de la valeur de la cote de retenue à cette date mais aussi des valeurs réalisées au cours de la période précédente : l'analyse précédemment décrite en $\S 5.2$. doit être perfectionnée. Nous ne rentrerons pas dans les détails de ce perfectionnement, mais retiendrons simplement que l'analyse statistique permet d'évaluer les caractéristiques des effets différés en écrivant simplement que la pression observée en un point résulte en réalité de la somme des effets de charges observés pour différentes tranches de temps ayaœnt précédé la mesure.

Cette analyse permet, bien que le régime permanent ne soit pratiquement jamais atteint, d'étudier de manière précise l'état hydraulique du noyau à retenue pleine en régime d'équilibre: l'ampleur des effets différés liés aux temps de propagation dans le matériau, à la suite des changements de cote de retenue amont, l'évolution dans le temps des pressions à conditions constantes de cote de retenue amont reconstituées.

\section{- Etat hydraulique moyen du noyau à retenue pleine}

La figure 15 a définit le comportement de la coupure dans la section située dans l'axe du sillon comme il apparaîtrait si la retenue amont était maintenant pleine pendant un temps assez long pour permettre à un équilibre de s'établir (théoriquement près de 200 jours, pratiquement 90 jours seulement et si la retenue aval était maintenue a la cote 650 pendant quelques jours. On observe que l'étanchéité est très homogène et qu'à retenue pleine la surface de saturation ne semble atteindre qu'à peine la zone de transition aval. 


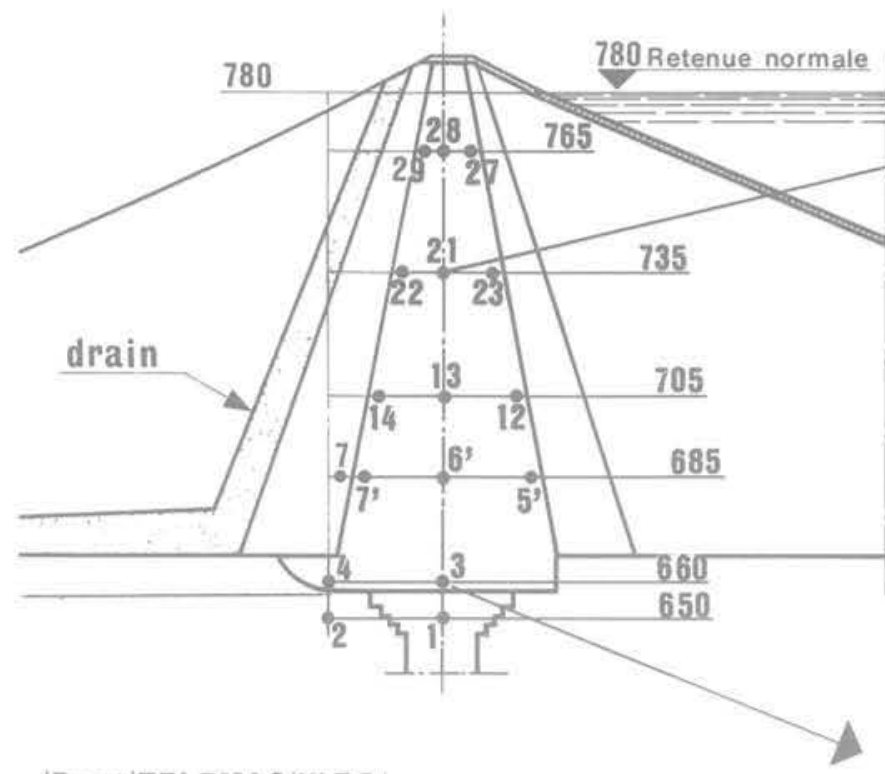

Repe.JTELEMAC W.E.S.
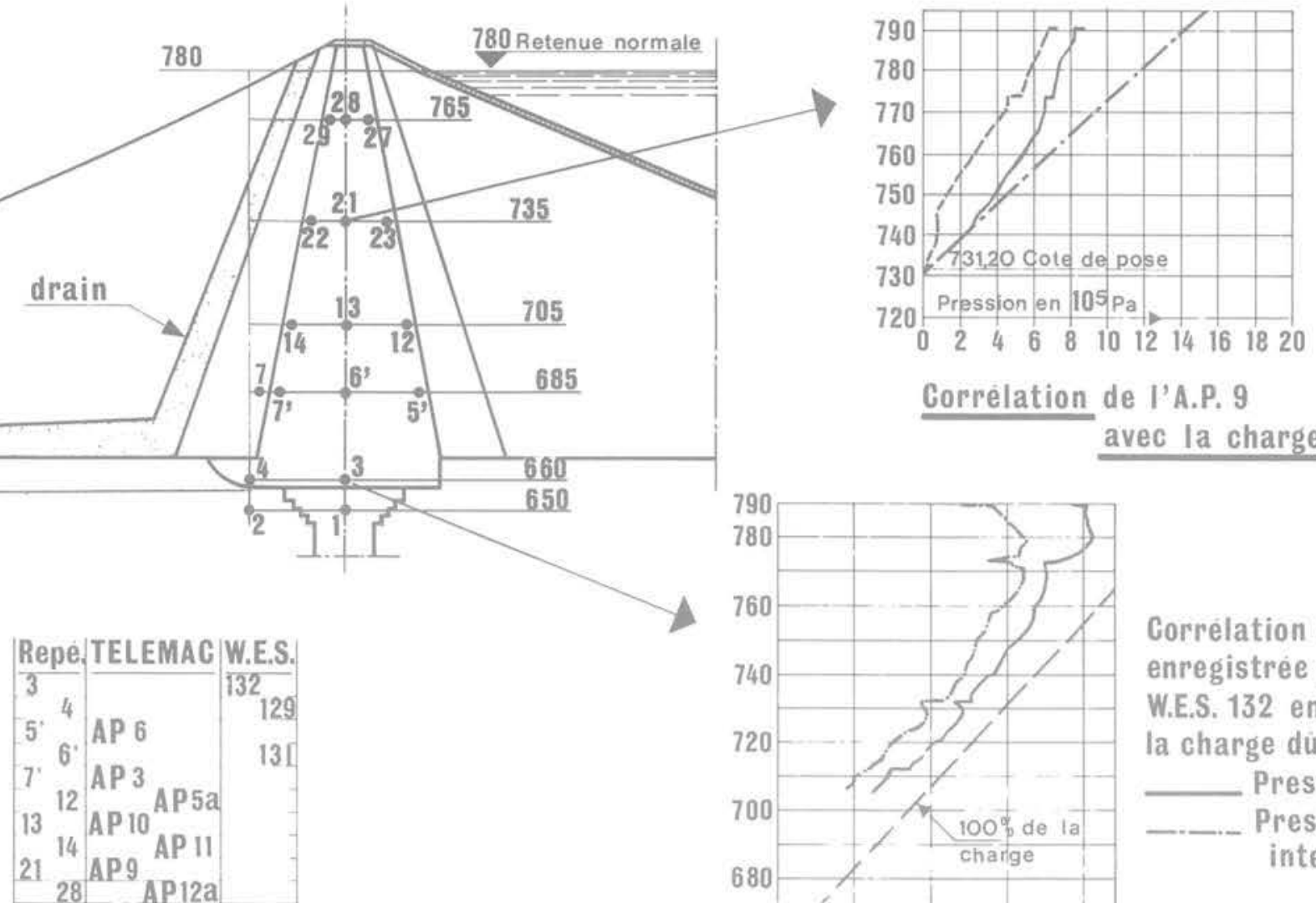

Correlation de I'A.P. 9

avec la charge

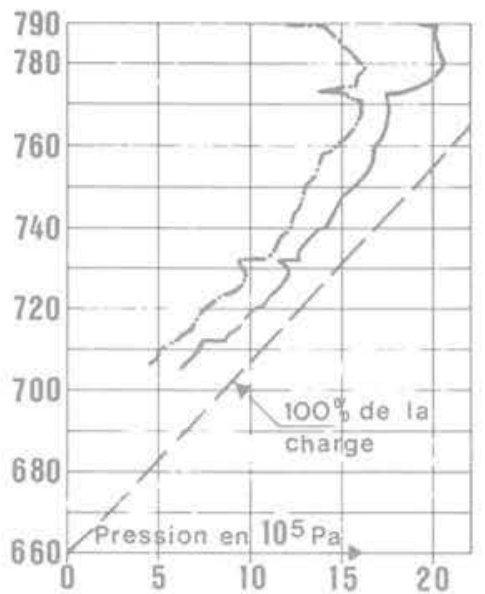

Correlation de la charge enregistree par la celulle W.E.S. 132 en fonction de la charge due au noyau Pressions totales Pressions intergranulaires

Fig. 10 Cellules de pressions totales

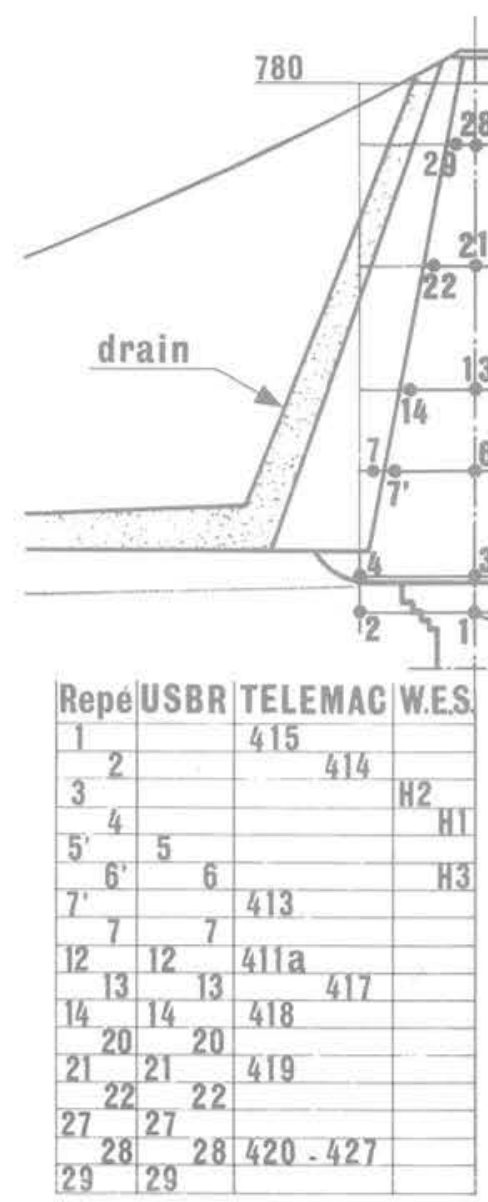

\begin{tabular}{|l|l|l|l|}
\hline U.S.B.R.6 & U.S.B.R. 13 & U.S.B.R. 21 & US.B.R.28
\end{tabular}
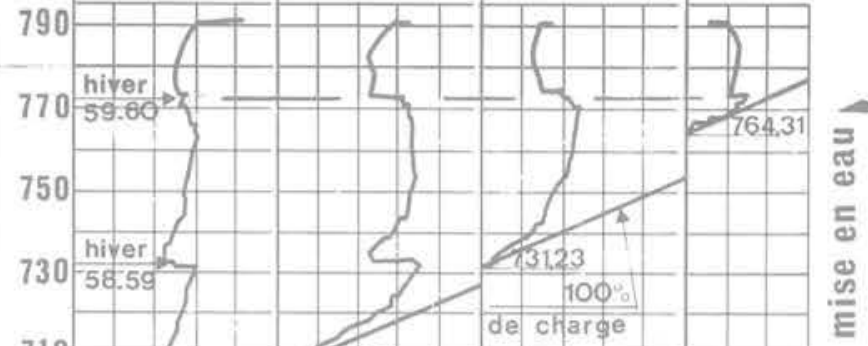

735

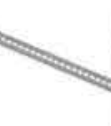

710

690
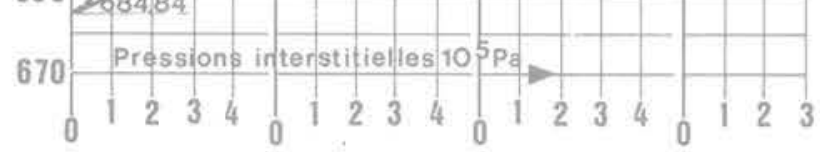

Correlation entre les cellules de l'axe de

la digue et la montee

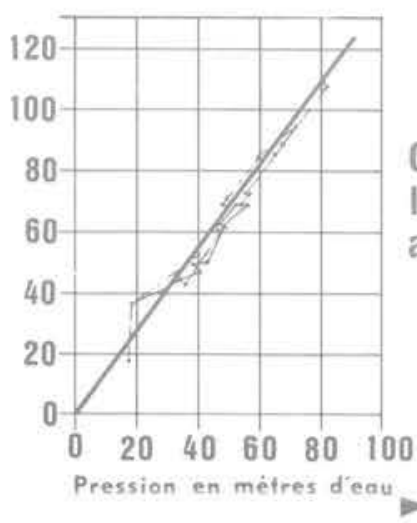

du noyau

Correlation de

la cellule 415

avec Ia mise en EAU

Fig. 11 Cellules interstitielles 


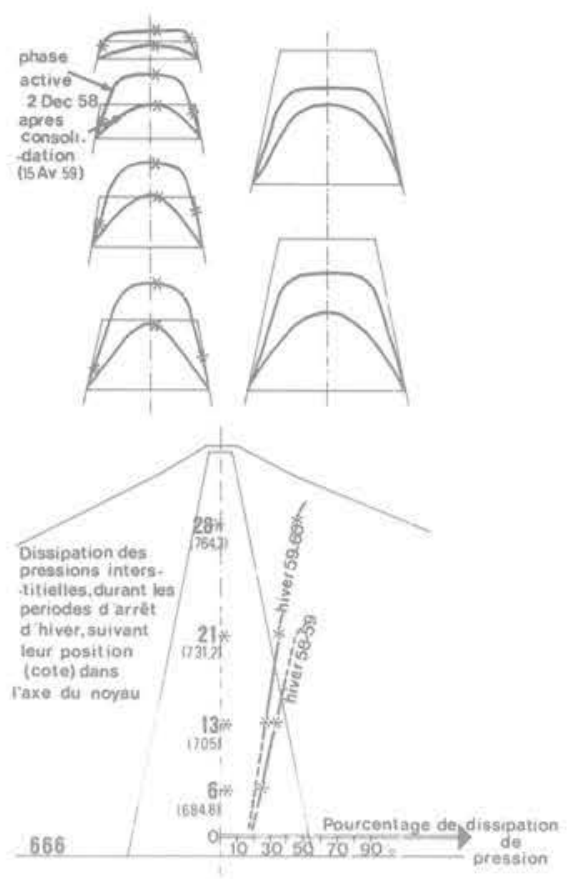

Fig. 12

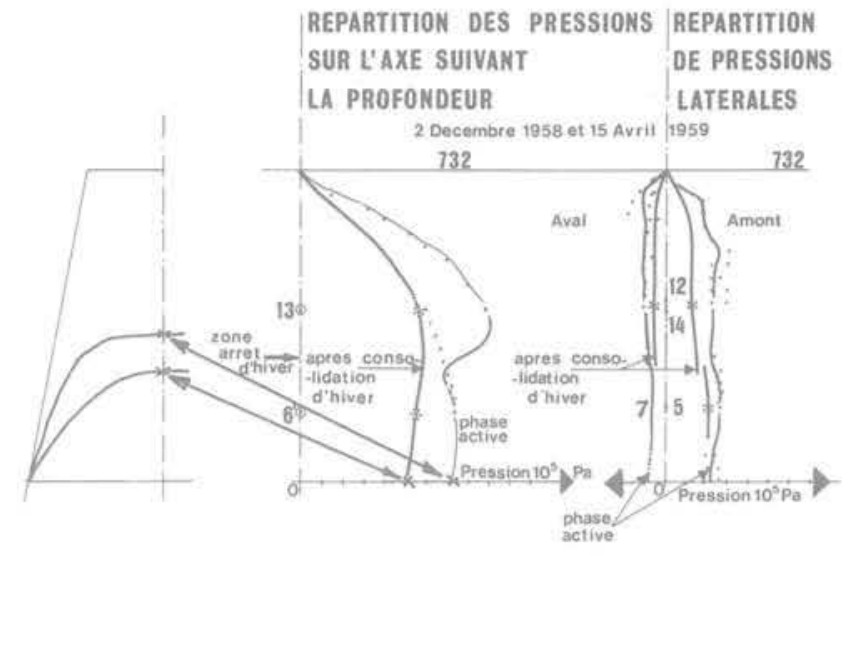

Fig. 13
REPARTITION deS PRESSIONS INTERSTITIELLES

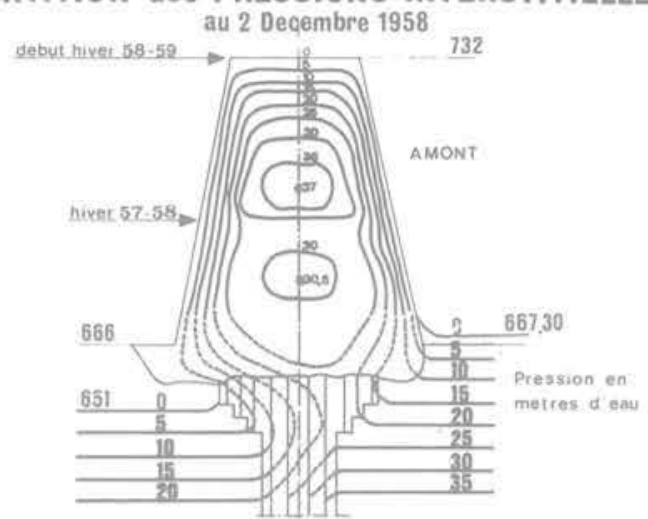

LIGNES EQUIPOTENTIELLES TUBES d'ECOULEMENTS au 2 Decembre 1958 au 2 Decembre 1958

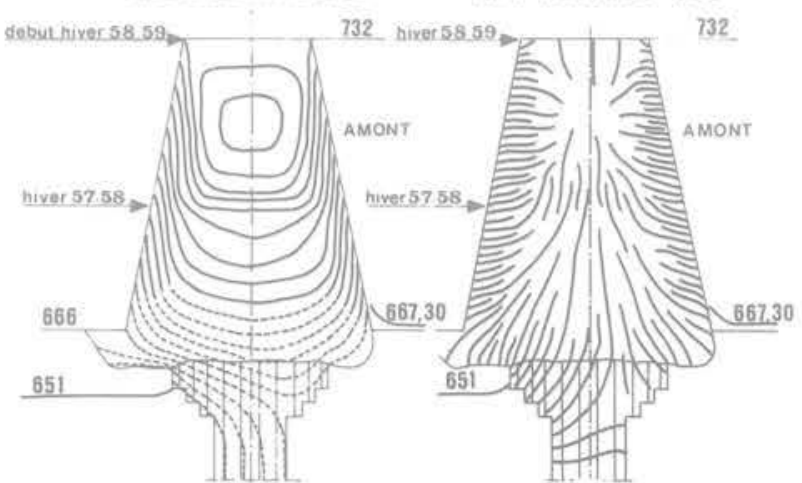

\section{REPARTITION deS PRESSIONS INTERSTITIELLES} au 15 Avril 1959

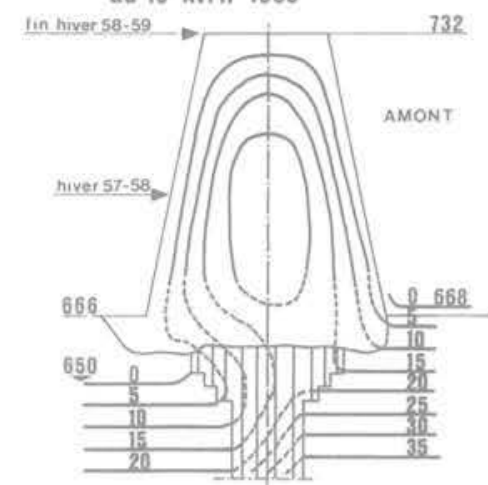

LIGNES EQUIPOTENTIELLES TUBES d'ECOULEMENTS

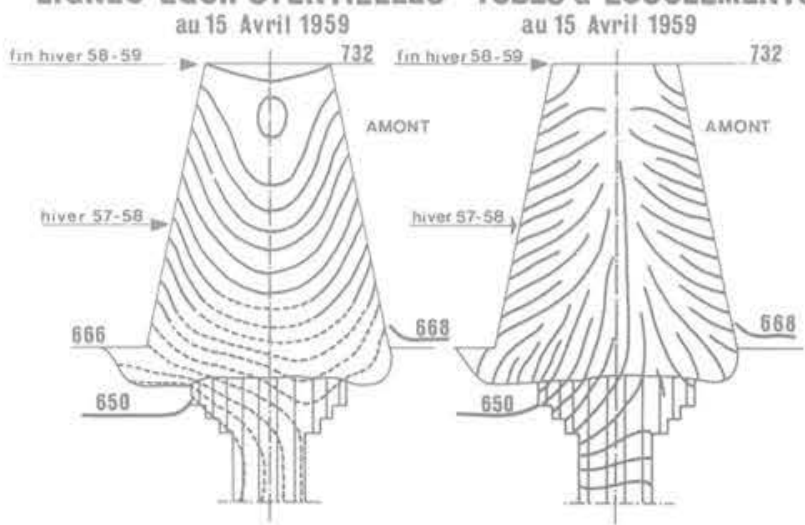

Fig. 14 

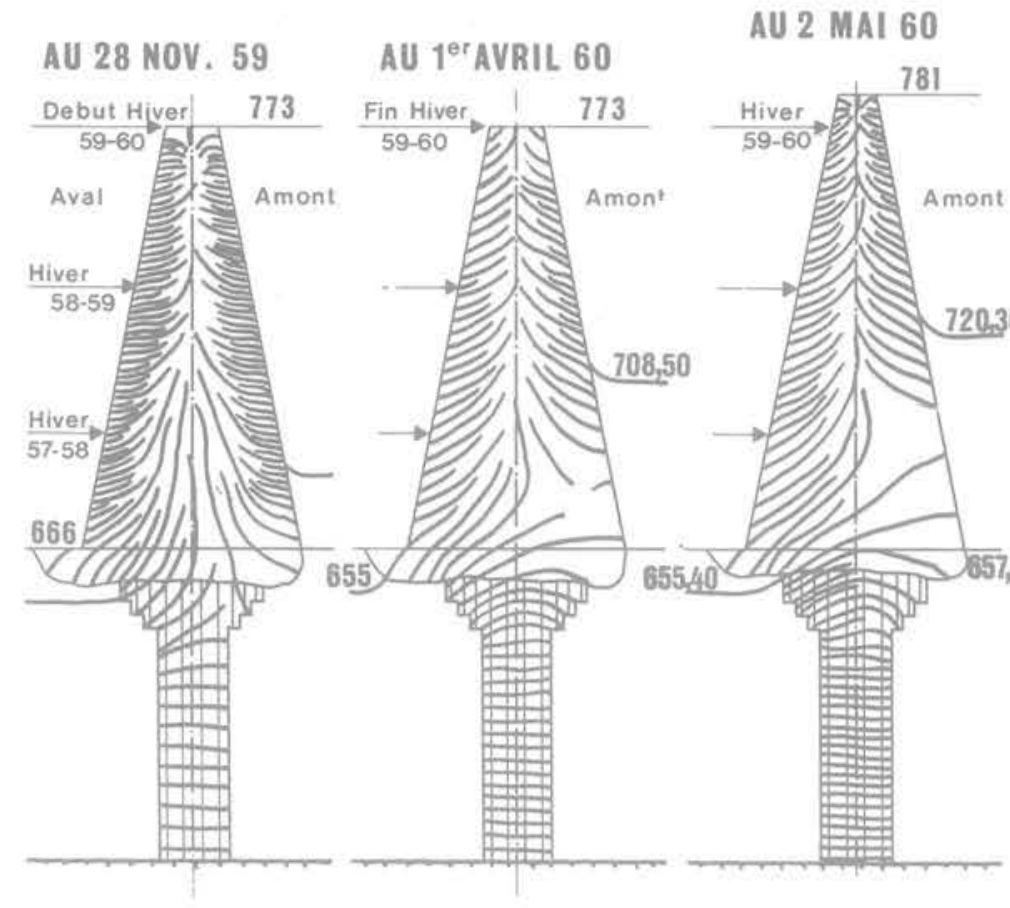

AU $1^{\text {er JUIN } 60}$

ECOULEMENTS DANS ET SOUS LA DIGUE
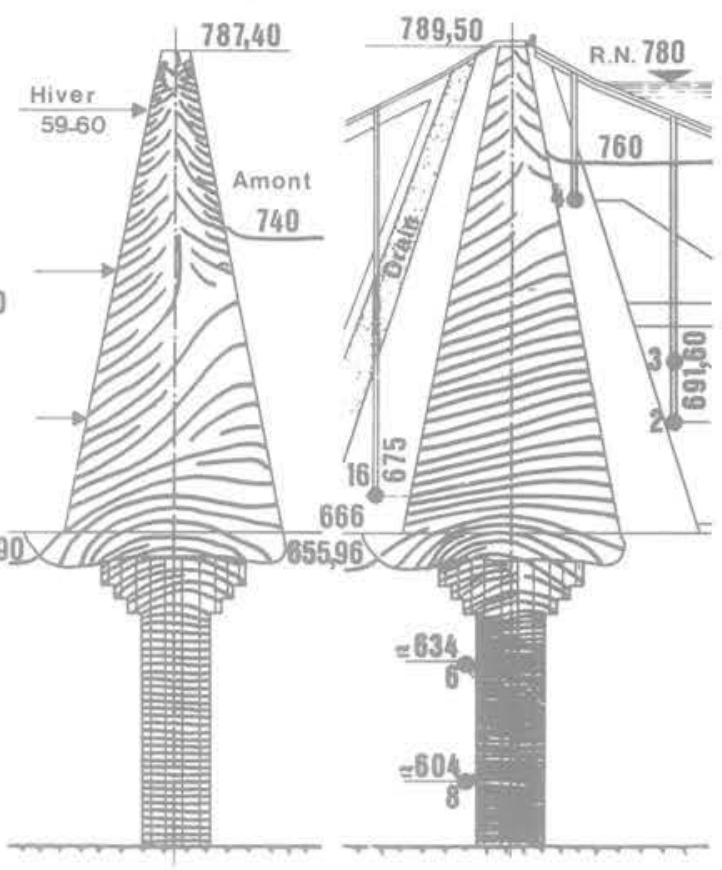

Egal débit lsaut coupure

$\mathrm{K}$ coupure $\neq \mathrm{K}$ noyau)

Fig. 15 Tubes d'écoulements pendant la mise en eau

Le débit de percolation à travers le noyau n'est collecté et mesuré que très imparfaitement et seulement sur la rive gauche sur une partie de la longueur sur laquelle il repose directement sur le rocher: il serait inférieur à $35 \mathrm{l} / \mathrm{mn}$ pour $250 \mathrm{~m}$ de digue dans une zone où elle a $100 \mathrm{~m}$ de hauteur.

- Réponse du noyau aux variations de la retenue amont

Les variations de charges consécutives aux variations de cote de retenue ne sont pas instantanées. La pression s'établit avec un retard de 5 à 10 jours à l'amont du noyau, de 5 à 120 jours dans l'axe, les retards étant plus faibles à la base: l'argile ne permet pas la transmission instantanée des charges et il y a contournement à la base par filtration rapide à travers les alluvions de fondation. Si l'on raisonne sur les états d'équilibre obtenus après un temps suffisant, on constate que les coefficients de proportionnalité des variations de charge et des variations de la cote de retenue sur les $40 \mathrm{~m}$ supérieurs sont pratiquement égaux aux charges observées en valeur relative (rapport de la charge observée à la diférence d'altitude du plan d'eau et de la cellule) : en première approximation la pression en un point du noyau varie linéairement avec la distance comptée verticalement entre le plan d'eau et ce point. La figure 16 résume les résultats obtenus en quelques points du noyau, tandis qu'un cycle annuel du fonctionnement est synthétisé sur la figure 17. On y voit l'état hydraulique du noyau à quatre dates, une année où la variation de cote de retenue est celle indiquée, très typique, de l'exploitation habituelle de cette retenue. Ces figures ont été établies en tenant compte des temps de réponse des diverses cellules. Leur examen appelle trois remarques :
- en Novembre, il coexiste simultanément une baisse de la charge à l'amont du noyau pratiquement en suivant le plan d'eau et plus à l'aval une certaine poursuite de l'accroissement des charges.

- pendant 9 mois de l'année, le noyau ne fait que restituer vers l'amont et surtout l'aval l'eau qu'il a accumulée pendant les trois autres mois pendant lesquels se remplissait la retenue.

- il permet de porter un jugement très satisfaisant sur le comportement moyen du noyau.

Les caractéristiques obtenues autorisent la détermination du comportement hydraulique du noyau pour toute variation prédéterminée du niveau amont; on peut ainsi porter un jugement sur les conséquences d'une exploitation particulière à l'avance.

\section{- Evolutions avec le temps}

Les effets réversibles des variations de cote de retenue étant bien définis tant sur le plan de leur ampleur que sur celui des retards, il est possible, après chaque mesure, de porter un jugement objectif sur l'évolution des caractéristiques hydrauliques du noyau quelles que soient les cotes réalisées au moment de la mesure et dans la période la précédant, c'est-à-dire à conditions constantes reconstituées : la connaissance précise du comportement normal du noyau rend donc la surveillance permanente très efficace: en fait, on peut affirmer qu'il n'y a eu aucune évolution significative du comportement hydraulique depuis 1961, voir sur la figure 18 la reproduction d'un graphique de surveillance d'une pression interne typique. 


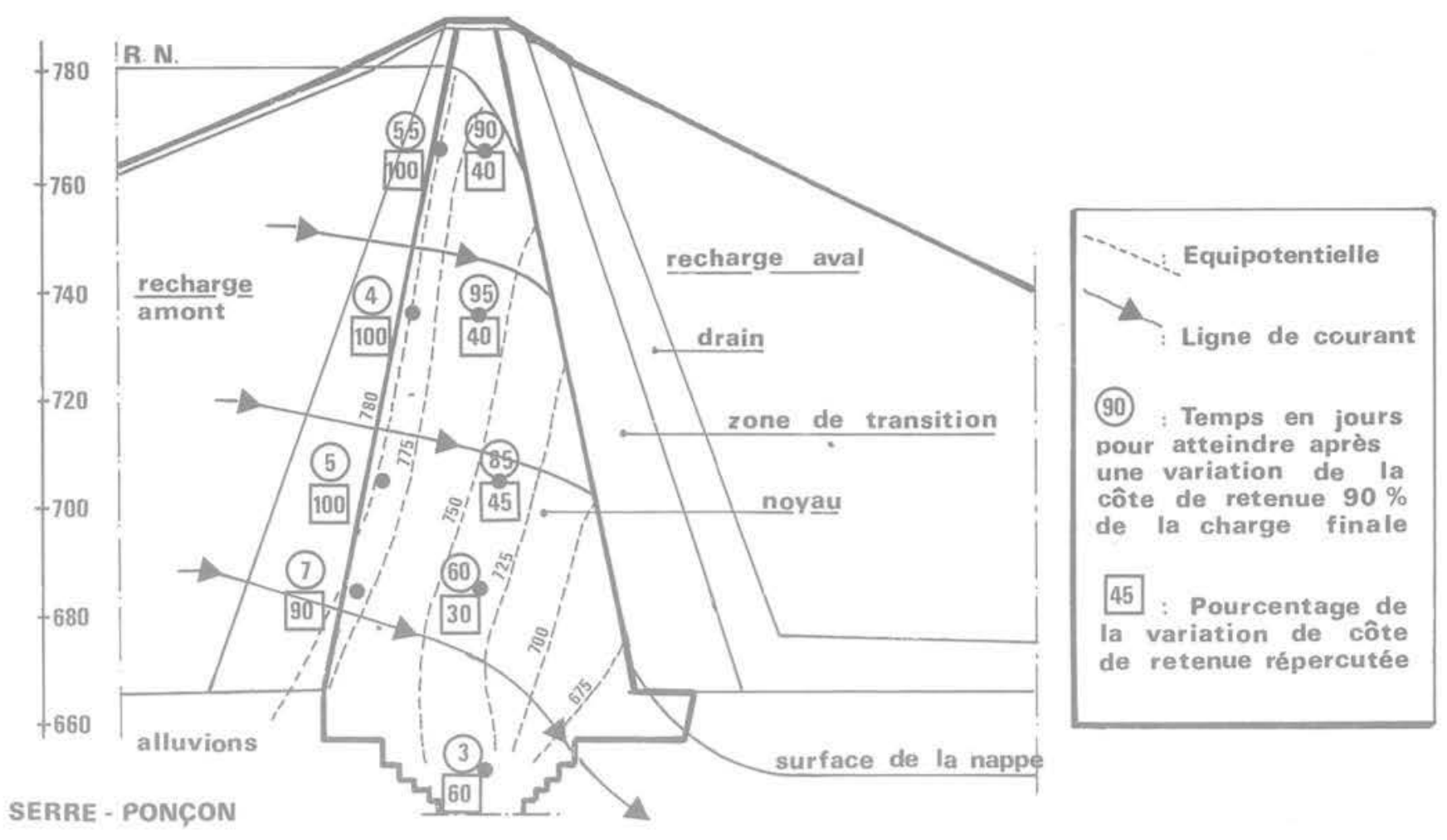

Fig. 16 Charge hydraulique dans le noyau
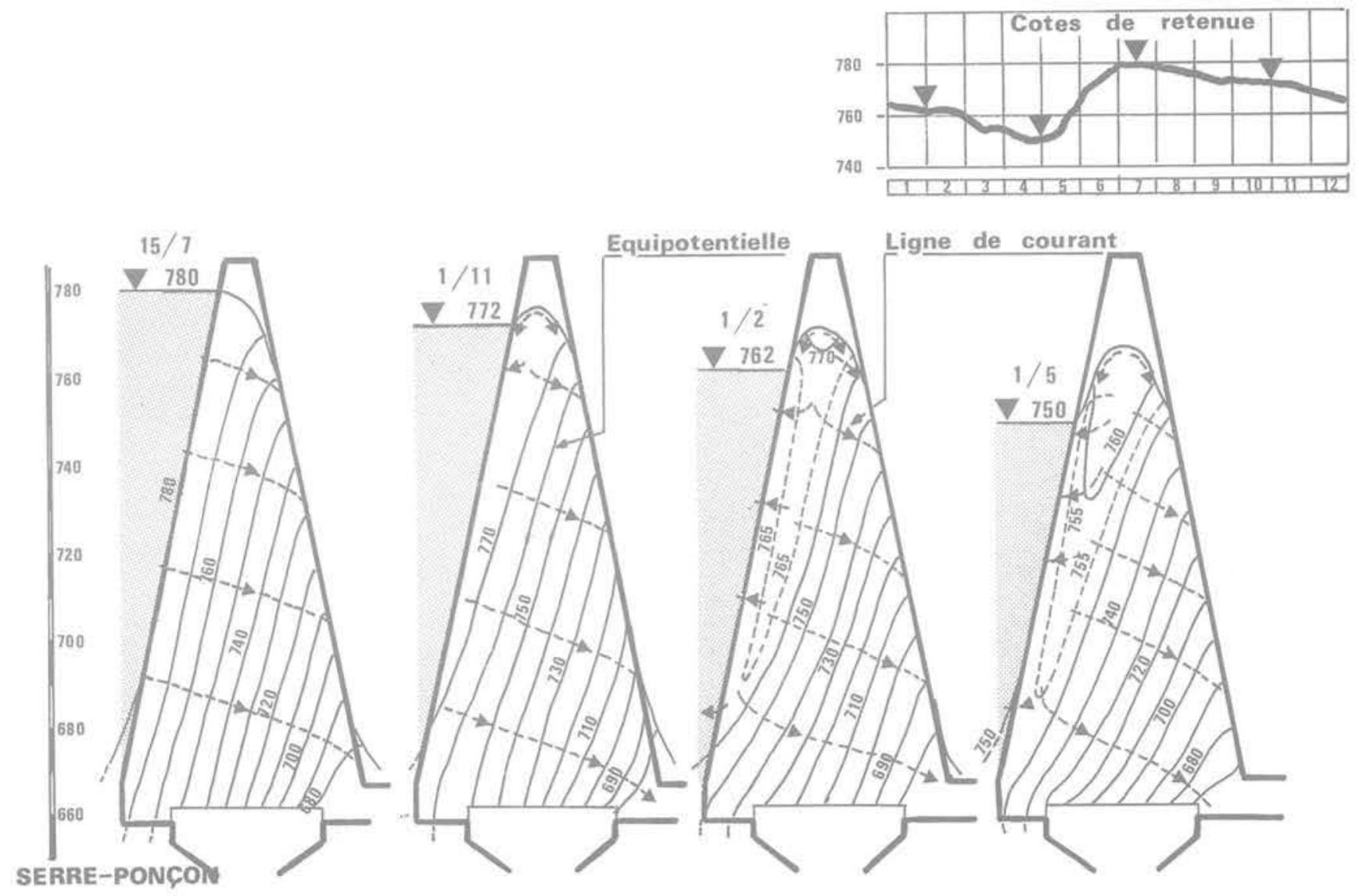

Fig. 17 Charge hydraulique dans les organes d'étanchéité pour le marnage annuel 
bars
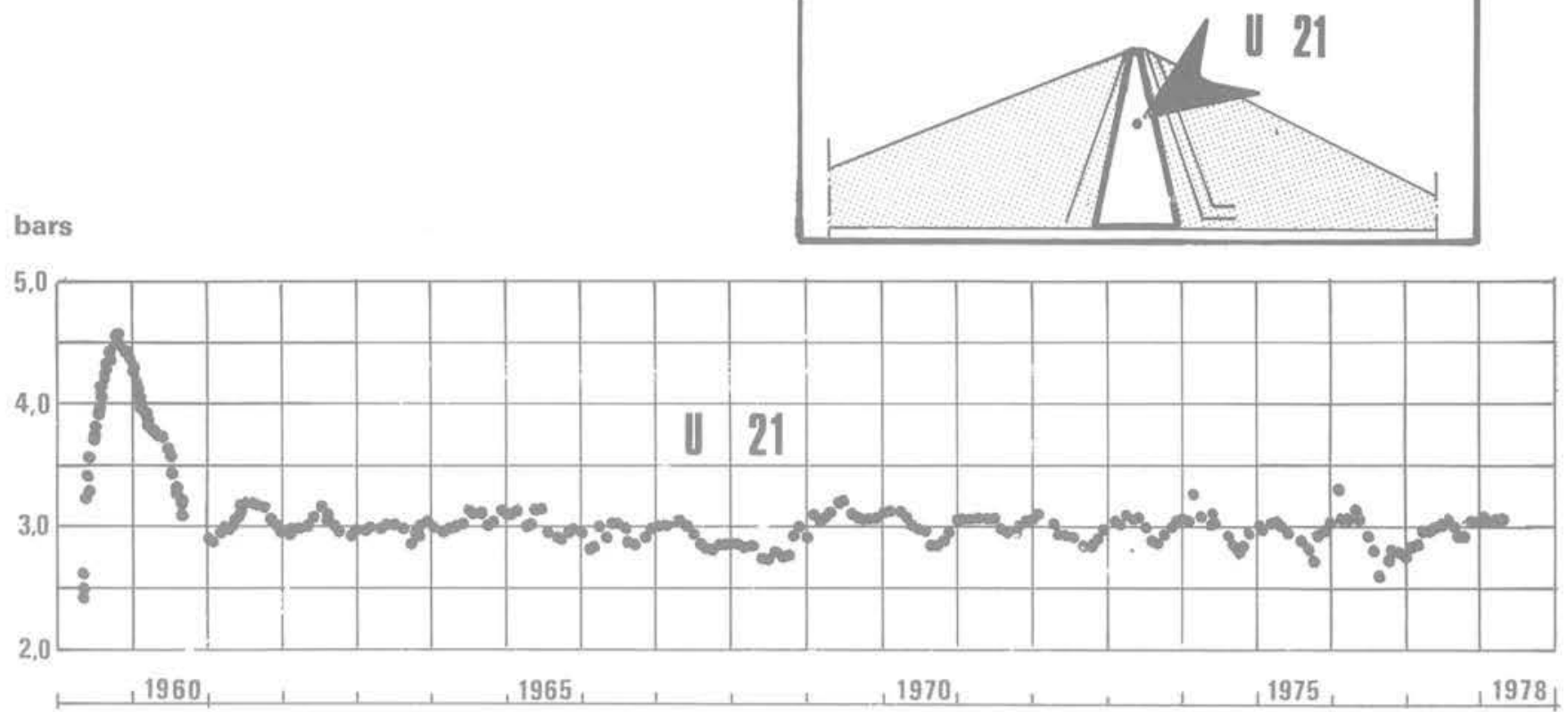

SERRE - PONÇON

Fig. 18 Pressions interstitielles sur l'axe du noyau

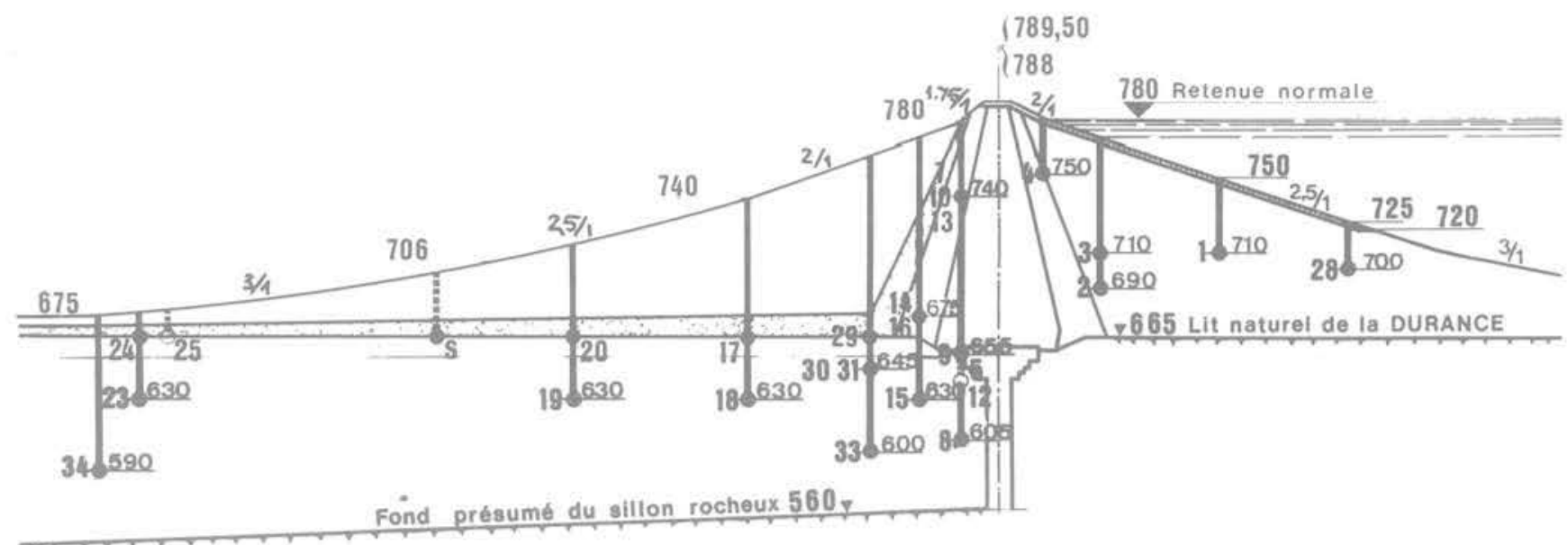

Fig. 19 Tubes piézométriques. Profil dans l'axe du sillon rocheux

\subsection{Les alluvions}

\section{- Alluvions des recharges}

Les alluvions de Durance sont souvent propres, de 2 à $5 \%$ d'éléments inférieurs à 80 microns, surtout extraites dans l'eau. Leur mise en place se fait à de fortes densités souvent supérieures à 2,3 et même 2,4 et malgré cela la perméabilité reste satisfaisante comme le prouvent les piézomètres amont (fig. 19). qui suivent les fluctuations de l'eau pendant la mise en eau ne conservant qu'une ligne piézométrique de très faible pente $4 / 1000$ au moins pour écouler l'eau stockée. II faut souligner que la mise en place à l'amont dans le sens aval-amont avec écrètement au bull a été favorable, créant une ségrégation favorisant les échanges d'eau entre la masse de la recharge et l'amont. La perméabilité moyenne calculée avec les piézomètres serait de l'ordre de $10^{-3}$ à $10^{-4} \mathrm{~m} / \mathrm{s}$.

\section{- Alluvions injectéos}

Lorsque ces alluvions ont été injectées afin de réaliser la coupure dite étanche, outre les nombreux essais d'eau. nous avons réalisé des puits visitables et observé un matériau bien injecté. Les premières mesures ont montré que cette coupure étanche a bien joué son rôle de séparation d'une nappe amont pratiquement horizontale (bien que peu d'instruments définissent les pressions juste en amont) et une nappe aval à faible pente, puisque plus de $95 \%$ de la charge est dissipée dans cette coupure de 15 mètres d'épaisseur théorique. Le gain de perméabilité pouvant être chiffré à 250 au minimum pour les parties homogènes et susceptibles de répondre à la loi de Darcy, la perméabilié obtenue devrait se situer vers $10^{-5} \mathrm{~m} / \mathrm{s}$. Les recoupements quant aux débits de percolation calculés (cf. exposé Comité de Mécanique du Sol, Novembre 1978 par M. Ledeuil, réf. 12) laissent penser que ce chiffre de $10^{-6}$ doit être divisé par 2 


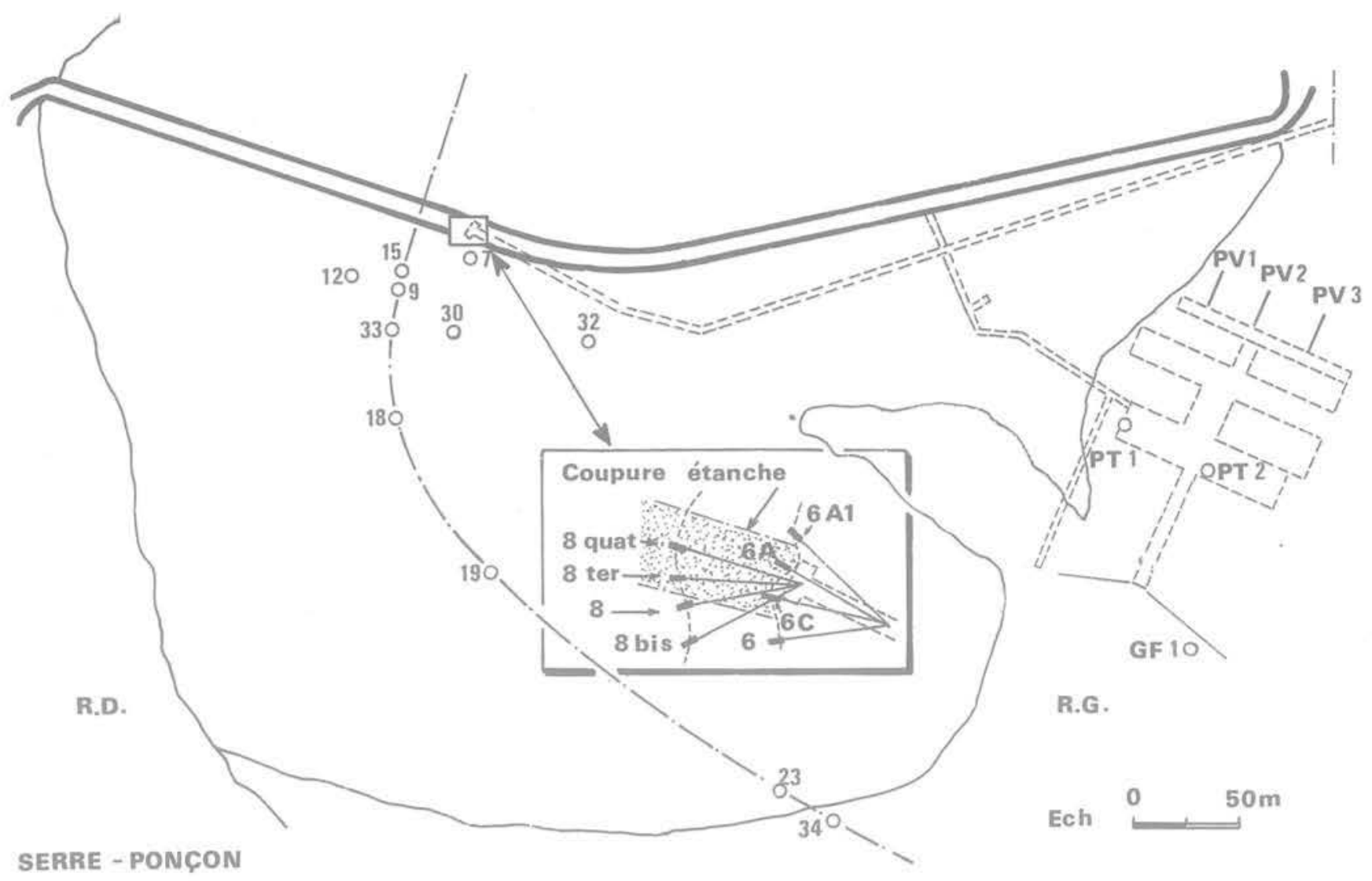

Fig. 20 Piézomètres. Piézomètres aval de la coupure étanche et de l'usine

ou 3. Les mesures poursuivies régulièrement depuis 1962 confirment que la géométrie de la zone injectée est probablement assez irrégulière (fig. 20) mais que globalement elle présente un fonctionnement conforme aux prévisions et sans aucune évolution notable depuis 1962. L'analyse des résultats a permis de chiffrer la différence entre les temps de réponse aux variations de charge dans la coupure et dans le noyau : cinq jours pour toute l'épaisseur de la zone injectée à comparer au retard de l'ordre de 100 jours introduit par le noyau. La manière différente dont les deux organes d'étanchéité jouent leur rôle est ainsi bien caractérisée.

\section{- Alluvions du sillon aval}

Les alluvions en place dans le sillon sont le siège de la nappe aval dont les fluctuations et l'évolution synthétisent la vie du barrage. Dans l'exposé cité au § 6.2. il a été calculé le débit global d'écoulement à travers le barrage et ses fondations, ce débit se retrouvant dans ce lit alluvial est suivi à l'aide des nombreux piézomètres décrits sur la figure 19. Au cours des sondages, plusieurs nappes superposées ont été détectées à $20 \mathrm{~m}$ de distance au bénéfice d'un horizon plus fin, la différence piézométrique faible est de l'ordre de 1 mètre ou moins. II arrive que cet état soit fugitif puis se reconstitue, l'horizon étanche jouant alors le rôle de soupape (cf. l'exposé cité en §̧6.2.). Transversalement de rive à rive une différence est également perceptible: l'écoulement va des rives au centre avec un niveau piézométrique peut être plus haut en rive droite. Toutes ces indications ou tendances sont intéressantes dans leurs évolutions car elles ont permis parfois d'expliquer des anomalies et ont guidé des traitements complémentaires telles les injections du rocher complétées après coup.

Le débit global trouvé lors de la première mise en eau soit 170 litres/seconde sous 110 mètres de charge ne semblait pas varier en fonction de la charge d'une façon linéaire. La loi de Darcy n'étant pas vérifiée, on pouvait considérer qu'une part du débit était partiellement concentrée. Depuis 1963, le débit semble avoir été divisé par 1,5 et la linéarité de la variation avec la charge presque atteinte (les mesures ne correspondent qu'à des variations du $1 / 3$ de la charge à partir du maximum, le barrage étant rarement vidé plus bas que la cote 740). Là encore, l'accumulation des mesures de niveau de la nappe correspondant à des niveaux variables de la retenue aval d'Espinasse (qui évolue constamment puisqu'il s'agit d'un bassin de compensation) a permis de préciser une des caractéristiques de son comportement: la réponse des cotes piézométriques aux variations de cote aval n'est pas instantanée et fait intervenir des retards qui ont pu être analysés comme précédemment.

Les retards s'échelonnent de l'aval à l'amont entre 5 et $70 \mathrm{~h}$. On peut indiquer que cette analyse n'est qu'un perfectionnement de celle qui avait été réalisée dès la mise en eau (voir l'article de M. Barge sous la référence 8). Elle donne la possibilité de vérifier à chaque instant, quelle que soit la cote de la retenue d'Espinasse, la pente de la nappe, donc son débit en relation directe avec l'efficacité hydraulique de la coupure. Aucune évolution significative n'a été notée depuis 13 ans (à condition constante de cotes amont et aval).

\subsection{Le rocher}

C'est un calcaire marneux franc, mais parfois fracturé, oủ des circulations d'eau importantes ont parfois été détectées ; des mesures au micro-moulinet dans certains forages ont mis en évidence l'existence de plusieurs nappes, la présence du trou favorisant des échanges importants de débits.

Après la mise en eau une campagne complémentaire d'injection a eu lieu pour épaissir la zone traitée au contact du noyau ou de la coupure étanche.

\section{- Pression en rive gauche}

Après ce traitement une dizaine de profils formés chacun de 3 (ou 5) piézomètres amont, médian et aval devaient permettre de vérifier que dans son ensemble le dispositif d'étanchéité fonctionne de manière très efficace depuis l'origine: (cf. fig. 21. et 22). 


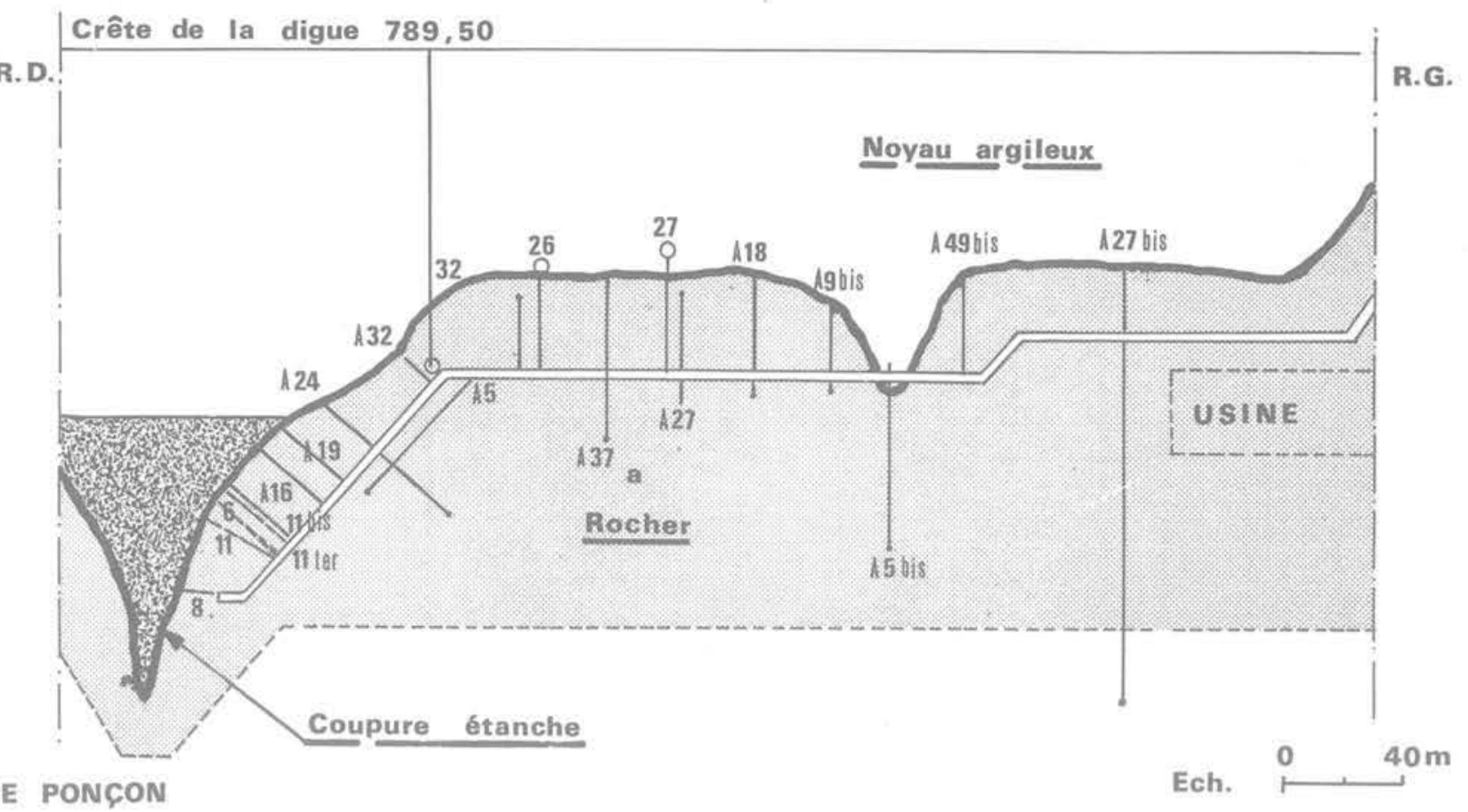

Fig. 21 Piézomètres. Situation du dispositif

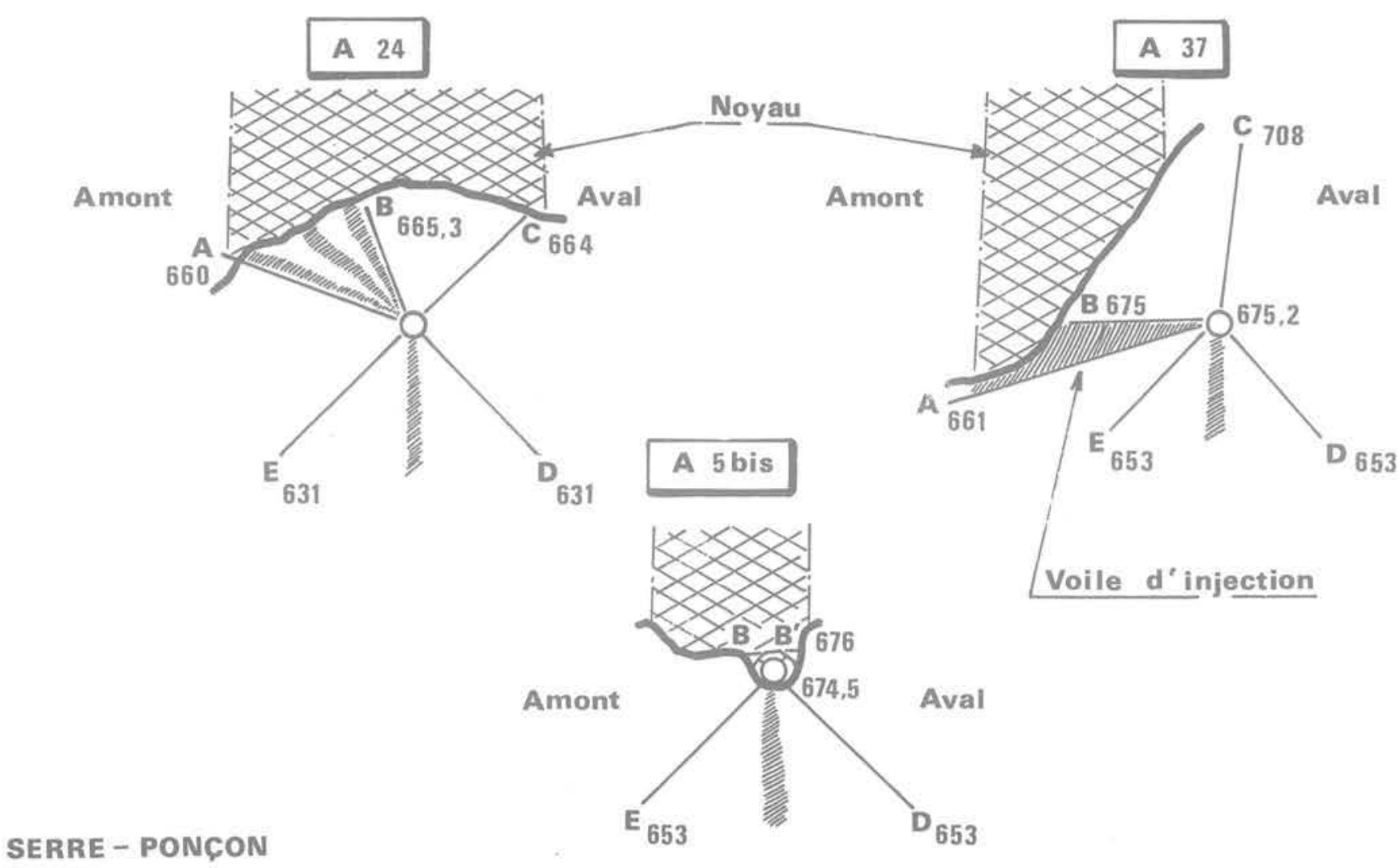

Fig. 22 Piézomètres. Auréoles de la rive gauche 


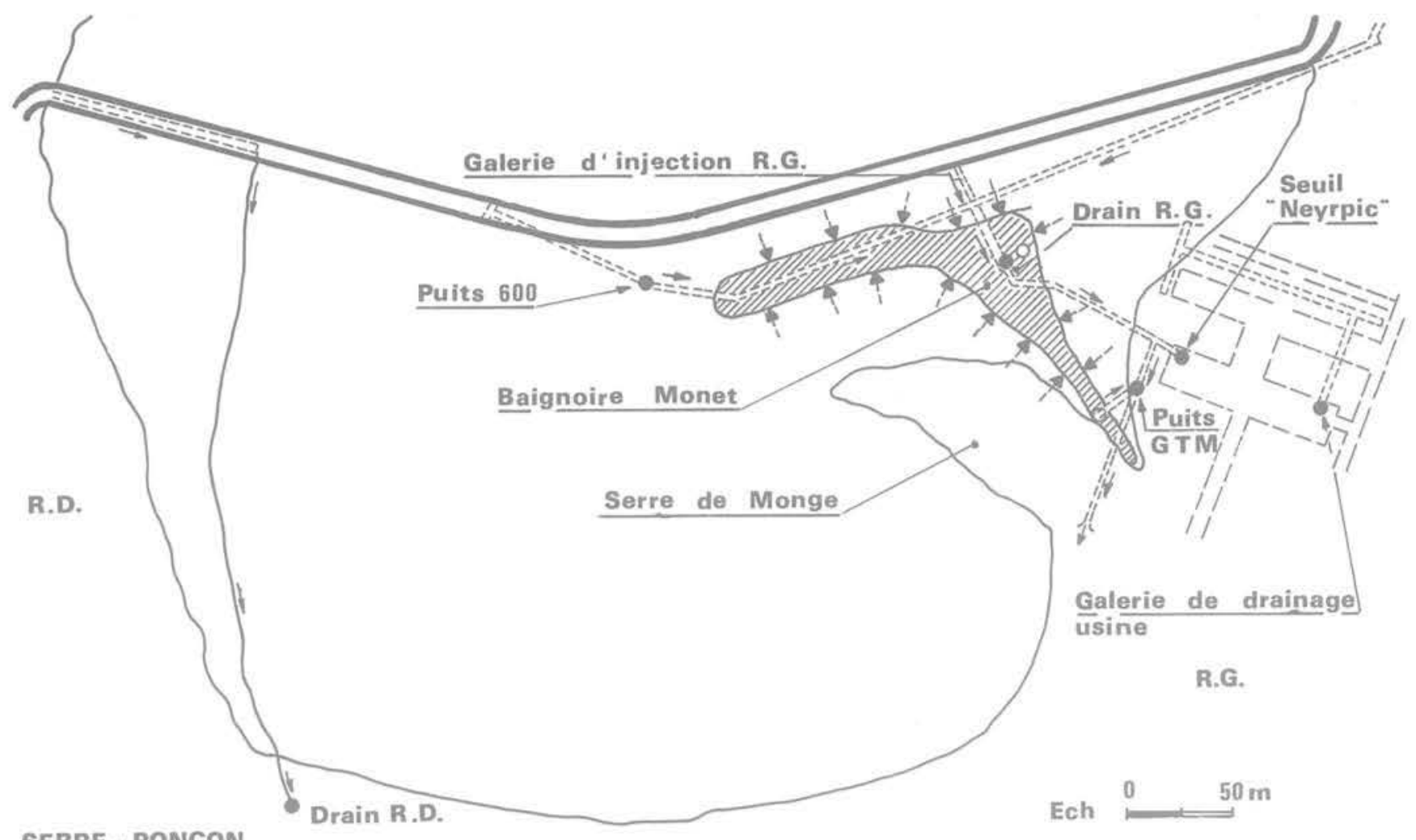

Fig, 23 Fuites. Situation du dispositif de mesure

\section{- En rive gauche}

Les charges évoluent de 90 à $60 \%$ en amont du noyau, à son pied et sous une certaine couverture de rocher ce qui semble indiquer une certaine imperméabilité du rocher en profondeur. A l'aval les pressions sont inférieures à $40 \%$ de la pression hydrostatique de la retenue. Elles passent à $30 \%$ dans la salle des transformateurs, $5 \%$ dans la salle des vannes, $0 \%$ sous la plate-forme d'accès à l'usine. La relation des pressions avec les variations de cote dans la retenue amont est étroite : le comportement est linéaire semble-t-il sur la totalité du marnage.

Les évolutions d'ensemble paraissent terminées depuis de nombreuses années : seules subsistent encore des variations locales à mettre en relation avec les particularités inévitables, à l'échelle des prises de pression, de la masse rocheuse.

\section{- Débits rive gauche}

Quant au débit de drainage de cette zone, il est difficile à analyser en détail, compte tenu de la complexité de la topographies et des particularités des dispositifs de collecte (cf. fig. 23). Dans la "galerie d'injection" qui est soumise à une charge d'eau comprise entre 100 à $140 \mathrm{~m}$, on recueille 6 à 7 $\mathrm{l} / \mathrm{mn}$ sur les $100 \mathrm{~m}$. situés sous le flanc gauche du sillon rocheux et 30 à $40 \mathrm{l} / \mathrm{mn}$ sur le reste $(400 \mathrm{~m}$ de longueur environ). Plus à l'aval, au droit de l'appui rive gauche de la digue les cavités de l'usine et leurs galeries de drainage sous une charge de $150 \mathrm{~m}$ recueillent $200 \mathrm{l} / \mathrm{mn}$.

Le volume total drainé et contrôlé ne représente naturellement qu'une partie de celui qui traverse les $90.000 \mathrm{~m}^{2} \mathrm{du}$ voile d'étanchéité et les $4.000 \mathrm{~m}^{2}$ de la coupure sous fluviale dont l'ordre de grandeur global peut être estimé à 10.000 $\mathrm{I} / \mathrm{mn}$. La modicité des fuites collectées en galerie avait déjà été soulignée aussitôt après la mise en eau à juste raison. Depuis, la relation entre débit et cote de retenue a pu être précisée : d'une manière générale les variations de débit sont proportionnelles aux variations de cote dans le 1/3 supérieur de la hauteur de retenue. Il est possible enfin de considérer comme hautement significatif du maintien de l'intégrité des caractéris-tiques de la coupue le fait qu'aucune évolution importante du débit n'est décelable depuis l'origine, hormis une décroissance du drainage de l'usine très régulière et du drainage du flanc gauche du sillon rocheux, maintenant très amortie.

\section{- Rive droite}

Le seul contrôle effectué est réalisé dans le drain R.D. oủ le débit est toujours très faible et non influencé par la retenue.

\section{Remarques sur le fonctionnement des dispositifs}

L'utilisation très régulière, depuis 20 ans, des appareils d'auscultation implantés sur le site du barrage de SerrePonçon et leur exploitation en vue d'assurer la surveillance permanente de l'ouvrage contribuent à enrichir l'expérience acquise sur les appareils et plus particulièrement sur leur capacité à fournir des résultats sûrs pendant une longue période. Les contrôles faisant appel aux techniques topographiques sont bien adaptés à ce type d'ouvrage où les parements à surveiller ont une grande surface et les mouvements significatifs sont d'ampleur relativement importante à comparer à ce que l'on peut attendre sur des ouvrages en béton similaires. II faut observer cependant que la précision obtenue en planimétrie ne peut être garantie qu'à la suite d'un contrôle minutieux et permanent des hypothèses de fixité des points sur lesquels s'appuie le réseau des stations d'oủ sont observés au théodolite les repères situés sur l'ouvrage, facilité par le respect d'une procédure rigoureuse dans l'exécution des mesures et l'utilisation de programme de calculs automatiques. Ceux-ci permettent de tirer le meilleur parti des visées réalisées de façon à réduire le plus possible l'effet des imprécisions de chacune et à déterminer les mouvements éventuels des piliers d'observation susceptibles de déplacements. En ce qui concerne les déplacements verticaux, leur détermination par des visées "zénithales" à partir du réseau d'observa- 
tion utilisé en planimétrie donne une précision très inférieure à celle que l'on peut obtenir á l'aide de niveau topographique de précision. Elle est cependant tout à fait suffisante pour les remblais de la taille de celui de SerrePonçon. L'utilisation de clinomètre dans des tubes verticaux s'est avéré inutilisable avec le matériel existant au moment du chantier pour déterminer les mouvements planimétriques internes avec une précision adaptée.

Il est probable que le matériel disponible actuellement surle marché permettrait de réal iser correctement une telle investigation. Cependant, la mise en place dans un noyau étanche de tubes susceptibles de devenir des conduits hydrauliques mettant en communication de zone à potentiel très différent, paraît à éviter dans toute la mesure du possible. La même remarque s'applique aux tubes des "cross-arms" destinés à contrôler les "tassements internes". Leur fonctionnement est satisfaisant à Serre-Poncon, bien qu'une rupture ait empêché d'utiliser la partie basse sur deux verticales au bout de 2 ans $1 / 2$ d'exploitation. Notons que la représentativité des mesures par rapport à la masse des remblais n'est jamais totalement assurée, puisque la mise en place nécessite un compactage particulier au droit de l'appareil.

Cellules de pressions interstitielles : des informations intéressantes ont pu être obtenues du fait que des cellules de type différent ont été associés en un même point (cf. tableau de la fig. 11)

Les cellules électriques à jauge de contrainte se sont avérées les moins fiables et ne donnent plus aucune indication utilisable. Les cellules à circuits hydrauliques ont donné satisfaction, mais il faut reconnaître que leur entretien nécessite de grandes précautions : les purges périodiques des fins et longs tubes de liaison ne doivent à aucun prix détériorer les interfaces cellule-terre.

Les cellules électriques á cordes vibrantes de Serre-Ponçon n'étaient pas compensées en température, ce qui a donné lieu à d'importantes incertitudes liées à la méconnaissance de leur température réelle de pose. Cependant, sur lonque période, elles donnent les résultats les moins dispersés et sont les plus pratiques d'emploi. Les matériels équivalents fabriqués actuellement sont compensés en température. Aucune expérience précise n'a été recueillie à SerrePonçon sur l'important problème de l'effet sur les cellules des périodes oú elles sont en zone non saturée.

Les cellules de charges totales à jauges de contrainte ou à corde vibrante n'ont pas été utilisées suffisamment longtemps pour qu'un jugement puisse être porté sur la qualité des résultats qui peuvent ètre fournis sur longue période. Les modèles utilisés à Serre-Ponçon semblent avoir donné des résultats plausibles au cours de la construction du remblai avec, cependant, une incertitude certaine liée à l'effet des variations de température et plus fondamentalement au principe mẻme de mesure faisant appel à une déformation: un résultat correct ne peut être obtenu qu'à la condition que la loi "charge-déformation" de l'appareil soit très proche de celle qu'au rait le matériau dont il prend la place. L'utilisation pour la surveillance à long terme de ce type d'appareil est d'un intérêt très réduit.

\section{Conclusion}

Le barrage de Serre-Ponçon a pu être construit dans de bonnes conditions, mis en eau sans dommages et exploité depuis vingt ans sans aucun signe de désordre apparent. A l'actif du dispositif d'auscultation. II faut indiquer qu'il a fourni des éléments essentiels au cours de ces trois phases:

- en assurant le contrôle du comportement des matériaux mis en place pendant la construction,

- en permettant la surveillance de la première mise en eau : il a permis de vérifier l'absence d'évolutions anormales dans les grandeurs contrôlées et la conformité globale aux prévisions,

- en apportant les éléments nécessaires à la compréhension du fonctionnement réversible, compréhension qui rend efficace pour le contrôle de la permanence de la sécurité, l'observation périodique des grandeurs mesu- rées; elle rend possible, après chaque mesure, la distinction des parts réversibles et irréversibles des variations observées, sur chacune desquelles peut être porté un jugement quant aux conséquences de leur poursuite pour la pérennité future de l'ouvrage. La constatation très systématique d'absence d'évolutions ou d'amortissements continus pousse aujourd'hui à émettre un jugement très favorable.

II faut également considérer qu'il a permis d'accroître la connaissance technologique des remblais en terre, précieuse pour l'optimisation des projets et constructions réalisés depuis ou à venir, sans compter l'expérience acquise sur les qualités des appareils eux-mêmes. Son rôle enfin n'est pas terminé puisqu'il contribuera durant de nombreuses années encore à assurer la surveillance, sans que l'on sache absolument, d'ailleurs, s'il est adapté à la détection dans des conditions acceptables sur le plan de la sécurité des populations et de l'économie du propriétaire, de tous les phénomènes qui seraient significatifs de désordre et de mise en danger à plus ou moins long terme. La répartition des phénoménes observés sur l'ensemble de l'ouvrage. mais aussi leur concentration suffisante en certaines zones, comme la section centrale du noyau autorisant une analyse du comportement, sans oublier la visite périodique intelligente de l'ouvrage par du personnel le connaissant bien, constituent des garanties sérieuses sur ce plan. Notons de ce point de vue l'importance fondamentale du contrôle des fuites, soit directement, soit par l'intermédiaire de la pente. de la nappe aval. L'évaluation, qui peut être faite, du montant de l'investissement consenti pour le matériel de mesure et sa mise en place, conduit à une valeur de l'ordre de $1 \%$ du prix de revient total de l'ouvrage. Les contraintes, que la mise en place et en service ont constitué pour le chantier. sont évidemment difficiles à apprécier : elles ne peuvent certainement pas être considérées comme négligeables.

La surveillance à court et moyen terme, assurée par le personnel sur place, occupe approximativement l'équivalent d'un agent technique à temps complet. Les mesures plus complexes, telles que celles faisant appel aux techniques de la topographie, le contrôle et la mémorisation de toutes les données recueillies au fur et à mesure de leur production, la tenue à jour des graphiques de surveillance en fonction du temps, de grandeurs significatives (déduites des mesures par des traitements appropriés), leur interprétation pour déceler des évolutions, en apprécier l'importance. porter un jugement sur le comportement futur de l'ouvrage, sont confiées en majeure partie à des équipes spécialisées disposant notamment des moyens de calculs sur ordinateur : cette tâche représente en moyenne chaque année 70 h d'ingénieurs; 600 h d'agents techniques, 20 h de calcul électronique. La dépense moyenne annuelle totale est de l'ordre de $300.000 \mathrm{~F}$, soit $0,05 \%$ de l'investissement. Voici quelques éléments du bilan qui peut être tiré aujourd'hui de l'expérience de vingt ans de mesures à Serre-Ponçon.

\section{Références Bibliographiques}

Parmi les nombreuses publications, nous pouvons citer :

[1] ISCHY, HAFFEN - Barrage de Serre-Ponçon, campagne de reconnaissance - Q 16, rapport 80 du $5^{\circ}$ Congrès des Grands Barrages Paris, 1955.

[2] MAIGRE - Réalisation d'un écran imperméable en matériaux alluvionnaires - Q 16, rapport 79 du $5^{\circ}$ Congrès des Grands Barrages Paris, 1955.

[3] SCHNEEBELI - Le barrage de Serre-Ponçon, Etude des infiltrations - Mémoire 19 Mars 1955.

[4] MAIGRE, BARGE - Compactage des matériaux utilisés pour la construction du noyau imperméable de la digue de Serre-Ponçon - Q 22, rapport 132 du $6^{\circ}$ Congrès des Grands Barrages New-York, 1958. 
[5] CABANIUS, MAIGRE - Aménagement de la Durance - Le Barrage de Serre-Ponçon - Revue Travaux Août 1958.

[6] LEDEUIL - Contribution à l'étude des contraintes, des déformations et des circulations d'eaux dans le barrage de Serre-Ponçon - Thèse de Doctorat Grenoble, 1960.

[7] Le Barrage de Serre-Ponçon

GUELTON, BALDY, MAGNE - Conception d'ensemble. O. de SCHNAKENBOURG - Contröle de l'exécution dela digue.

BARGE - Exécution des travaux - Revue Travaux Mai 1961 consacrée au $7^{\circ}$ Congrès International des Grands Barrages Rome, 1961.

[8] L'auscultation de la digue de Serre-Ponçon - Annales de I'Institut du Bâtiment et des Travaux Publics - Sept. 1963.
[9] BARGE, POST, HUYNH - Auscultation de la digue de Serre-Ponçon - Q 29, rapport 3 du $8^{e}$ Congrès des Grands Barrages Edimbourg, 1964.

[10] E.D.F., Division Technique Générale de la Production Hydraulique - Rapports d'auscultation de Serre-Ponçon : $N^{\circ} 1(1961-64) N^{\circ} 2(1965-67) N^{\circ} 3(1968-71) N^{\circ} 4(1972-74)$ $N^{\circ} 5(1974-76) N^{\circ} 6(1977-78)$.

[11] POST, LONDE "Les barrages en terre compactée" page 174, Gauthier - Villars, Edition 1953.

[12] LEDEUIL - Estimation du débit et de la nature des fuites d'un barrage en terre sur lit alluvionnaire (Application Serre-Ponçon). Exposé fait au C.F.M.S. le 13 Novembre 1978. 